\title{
Article \\ Frequency Contour-Strip Method for Characterization of Damage in Structures under Noisy Conditions
}

\author{
Jiayi Peng ${ }^{1,2}$, Hao Xu ${ }^{3}$, Hailei Jia ${ }^{1}$, Dragoslav Sumarac ${ }^{4,5}$, Tongfa Deng ${ }^{4}$, Xin Zhang ${ }^{1}$ (i) and Maosen Cao ${ }^{1,4, *}$ \\ 1 College of Mechanics and Materials, Hohai University, Nanjing 210098, China; pjiayi@126.com (J.P.); \\ jhl1024@sina.com (H.J.); zhangxinhhu@163.com (X.Z.) \\ 2 State Key Laboratory of Safety and Health for In-Service Long Span Bridges, Jiangsu Transportation Research \\ Institute, Nanjing 211000, China \\ 3 State Key Laboratory of Structural Analysis for Industrial Equipment, Faculty of Vehicle Engineering and \\ Mechanics, School of Aeronautics and Astronautics, Dalian University of Technology, Dalian 116024, China; \\ xuhao@dlut.edu.cn \\ 4 Jiangxi Province Key Laboratory of Environmental Geotechnical Engineering and Hazards Control, \\ Jiangxi University of Science and Technology, Ganzhou 341000, China; dragosumi@gmail.com (D.S.); \\ dbdtf@163.com (T.D.) \\ 5 Department of Technical Sciences, Civil Engineering, State University of Novi Pazar, 36300 Novi Pazar, Serbia \\ * Correspondence: cmszhy@hhu.edu.cn; Tel.: +86-1525-184-1008
}

Citation: Peng, J.; Xu, H.; Jia, H.; Sumarac, D.; Deng, T.; Zhang, X.; Cao, M. Frequency Contour-Strip Method for Characterization of Damage in Structures under Noisy Conditions. Appl. Sci. 2021, 11, 11479. https:// doi.org/10.3390/app112311479

Academic Editor: Giuseppe Lacidogna

Received: 4 October 2021

Accepted: 18 November 2021

Published: 3 December 2021

Publisher's Note: MDPI stays neutral with regard to jurisdictional claims in published maps and institutional affiliations.

Copyright: () 2021 by the authors. Licensee MDPI, Basel, Switzerland. This article is an open access article distributed under the terms and conditions of the Creative Commons Attribution (CC BY) license (https:// creativecommons.org/licenses/by/ $4.0 /)$.

\begin{abstract}
Eigen-frequency, compared with mode shape and damping, is a more practical and reliable dynamic feature to portray structural damage. The frequency contour-line method relying on this feature is a representative method to identify damage in beam-type structures. Although this method has been increasingly applied in the area of damage identification, it has two significant deficiencies: inefficiency in establishing the eigen-frequency panorama; and incompetence to identify cracks in noisy conditions, considerably impairing the effectiveness in identifying structural damage. To overcome these deficiencies, a novel method, termed the frequency contour-strip method, is developed for the first time. This method is derived by extending the frequency contour line of $1 \mathrm{D}$ to frequency contour strip of 2D. The advantages of the frequency contour-strip method are twofold: (i) it uses the isosurface function to instantly produce the eigen-frequency panorama with a computational efficiency several orders of magnitude higher than that of the frequency contour-line method; and (ii) it can accommodate the effect of random noise on damage identification, thereby thoroughly overcoming the deficiencies of the frequency contour-line method. With these merits, the frequency contour-strip method can characterize damage in beam-type structures with more efficiency, greater accuracy, and stronger robustness against noise. The proof of concept of the proposed method is performed on an analytical model of a Timoshenko beam bearing a crack and the effectiveness of the method is experimentally validated via crack identification in a steel beam.
\end{abstract}

Keywords: eigen-frequency; crack detection; frequency contour-line method; frequency contour-strip method; noise robustness

\section{Introduction}

Damage in a structure, e.g., a crack, induces local flexibility [1] that further alters structural dynamic characteristics [2-7], typically structural modal parameters including eigenfrequency, mode shape, and damping [8]. On this basis, the alteration of modal parameters forms the philosophy of vibration-based damage identification in structures [9-13]. Up to date, eigen-frequency, mode shape, or damping has been adopted for the detection of structural damage [14-18]. In practice, the acquisition of mode shape needs dense and numerous measurement points or expensive local-area sensing device such as scanning laser vibrometer and the evaluation of damping is an intractable operation due to existence of multiple influential factors $[19,20]$. In contrast, the eigen-frequency is more suitable for damage characterization because of its facilitation in acquiring and robustness to noise [21]. Eigen-frequency is a 
fundamental and reliable feature to portray damage from a practical perspective. Various eigen-frequency-based methods can be found in applications to damage identification in the literature [22,23]. In particular, the frequency contour-line method has gained more attention in the field of structural damage identification in recent years [24-28]. Identifying damage based on the frequency contour-line method involves five components: eigen-frequency panorama, experimental eigen-frequencies, frequency contour lines, frequency projection lines, and intersection point indicating damage.

The representative methods of using frequency contour-line method to detect the damage in a structure are briefed as follows. Dahak et al. [29] proposed a contour-line method on the identification of the damage location and depth in a beam, using only the value of the changes in the measured natural frequencies and the vectors of the curvature mode shapes of the intact structure. The effectiveness of the method was correctly verified in numerical and experimental cases. Banerjee et al. [30] presented two techniques to detect damage in a beam with functionally graded materials, one using frequency contour-line method and the other using a genetic algorithm optimization technique. The crack detection techniques and the numerical analysis showed good agreement. Yang et al. [31] introduced a contour-line method based on only post-damaged eigen-frequencies to estimate damage parameters. The capability of the method was demonstrated by the numerical and experimental results. Li et al. [32] utilized a wavelet finite element method to obtain the changes of natural frequency of a structure with multiple cracks and predicted the location and depth using the frequency contour-line method. The accuracy of the proposed method was fully verified. Mazanoglu and Sabuncu [33] improved the contour-line method through an algorithm, making it capable to locate the suitable positions of two cracks. Satisfactory location of the cracks was achieved. Chen et al. [34] presented a multiresolution finite element method capable of modal analysis for singularity problems and then plotted the first three calculated natural frequencies contour line to predict the damage, with the cracks quantitative identification method experimentally validated. Cao et al. [35] conducted the performance assessment of the frequencies contour-line method in characterizing cracks in noisy conditions, a guideline for rational use of natural frequencies to identify cracks in actual beam-type structures provided. Kalanad and Rao [36] proposed a methodology to detect cracks in conjunction with improved cracked element for singularity problems. This methodology efficiently identified crack location and size based on the contour-line method. Swanidas et al. [37] considered the vibration of cracked beams using both Timoshenko and Euler formulations. The frequency contour-line method identified the crack size and location properly, using the lower order frequencies. Yu and Chu [38] developed a p-version of finite element method to determine the location and size of an open edge crack in functionally graded material (FGM) beams and used the contour-line method to identify the crack parameters. The performance of the method was demonstrated efficiently by numerical experiments. Lakshmanan et al. [39] proposed a contour-line method for damage identification from measured natural frequencies of a contiguously damaged reinforced concrete axial rod and beam, with the pros and cons of this method proved by experiments. Dong et al. [40] applied the contour-line method in a static rotor with an open crack, based on the assumption that the cracked rotor was an Euler-Bernoulli beam with circular cross-section. Two experimental examples demonstrated the availability of the method. Liu et al. [41] presented a contour-line method for identification in a breathing cracked beam, combined with the natural frequency equation and stiffness model. The feasibility of this method was verified. The above studies demonstrate that the frequency contour-line method functions as an active research branch in structural damage identification.

Despite the prevailing of the frequency contour-line method, two remarkable deficiencies in this method can be observed in damage detection: (i) inefficiency in establishing the eigen-frequency panorama and (ii) incompetence to identify cracks in noisy conditions. These shortcomings fairly impair the efficiency, accuracy, and robustness of the method in identifying damage. To address these deficiencies, a more sophisticated method, termed frequency contour-strip method, is developed in this study. The frequency contour-strip 
method is developed based on promoting the contour-line method by extending the line to a strip, and the strip endows the new method with more efficiency, greater accuracy, and stronger robustness to noise. The proof of concept of the method is performed on an analytical model of a cracked Timoshenko beam and the method is experimentally validated on crack identification in a steel beam.

The remainder of this paper is organized as follows. Section 2 presents the analytical model for transverse vibration of Timoshenko beam bearing a crack. Section 3 introduces the frequency contour-line method with its limitations in crack identification clarified. Section 4 develops the frequency contour-strip method with emphasis on its advantages over the frequency contour-line method. Section 5 validates the method via an experiment on detecting cracks in a beam. Conclusions are given in Section 6.

\section{Transverse Vibration of Timoshenko Beam Bearing a Crack}

A cracked cantilever beam with a uniform rectangular cross-section of length $L$, width $B$, and thickness $H$ is shown in Figure 1 where $\mathrm{w}$ and $\mathrm{v}$ denote the vertical and horizontal coordinates, respectively. $x$ and $y$ denote the dimensionless crack location and depth, respectively. The Timoshenko theory is used to establish the governing differential equation for transverse vibration of the beam, given as

$$
\begin{gathered}
E I \frac{\partial^{2} \psi(v, t)}{\partial v^{2}}+k G D\left(\frac{\partial w(v, t)}{\partial v}-\psi(v, t)\right)-\rho I \frac{\partial^{2} \psi(v, t)}{\partial t^{2}}=0 \\
k G\left(\frac{\partial^{2} w(v, t)}{\partial v^{2}}-\frac{\partial \psi(v, t)}{\partial v}\right)-\rho \frac{\partial^{2} w(v, t)}{\partial t^{2}}=0
\end{gathered}
$$

where $w(v, t)$ and $\psi(v, t)$ are the transverse deflection and rotation angles at the location $v$ in the $v$ axis and time $t$, respectively; $E$ and $G$ are the elastic modulus and shear modulus, respectively; $I, \rho$, and $D$ are the area moment of inertia, mass density, and cross-sectional area, respectively; and $k$ is the shear coefficient.
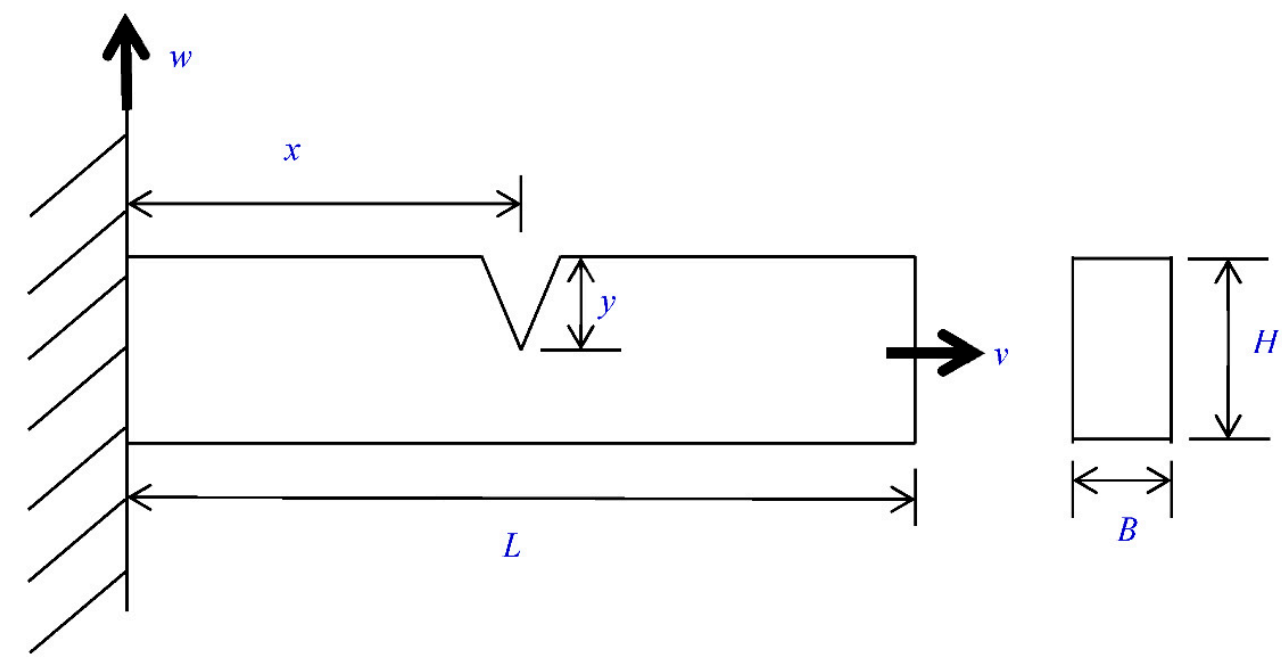

Figure 1. A cantilever beam with a crack.

\subsection{Intact Beam}

Assuming the beam is undamaged, the general solutions of Equations (1a) and (1b) can be derived based on the method of separation of variables as $w(v, t)=W(v) T(t)$ and $\psi(v, t)=\Psi(v) T(t)$, where $T(t)$ is only a function of time t. $W(v)$ and $\Psi(v)$ are given as follows:

$$
W(v)=A_{1} \cosh \lambda_{1} v+A_{2} \sinh \lambda_{1} v+A_{3} \cos \lambda_{2} v+A_{4} \sin \lambda_{2} v
$$




$$
\Psi(v)=A_{2} m_{1} \cosh \lambda_{1} v+A_{1} m_{1} \sinh \lambda_{1} v-A_{4} m_{2} \cos \lambda_{2} v+A_{3} m_{2} \sin \lambda_{2} v
$$

where $A_{i}(i=1-4)$ are constants to be determined with the boundary conditions; $\lambda_{1}, \lambda_{2}, m_{1}, m_{2}$, are defined, respectively, as

$$
\begin{aligned}
& \lambda_{1}=\left(\sqrt{a^{2}-b}-a\right)^{1 / 2}, \lambda_{2}=\left(\sqrt{a^{2}-b}+a\right)^{1 / 2}, m_{1}=\frac{\tau s+\lambda_{1}^{2}}{\lambda_{1}}, m_{2}=\frac{\tau s-\lambda_{2}^{2}}{\lambda_{2}}, \\
& a=\frac{\tau(r+s)}{2}, b=\tau(\tau r s-1), r=\frac{I}{D L^{2}}, s=\frac{E}{k G} r, \tau=\frac{\rho D}{E I} L^{4} \omega^{2},
\end{aligned}
$$

with $\omega$ being the eigen-frequency. For a certain beam, the geometric and physical parameters are all given. Therefore, $\lambda_{1}, \lambda_{2}, m_{1}, m_{2}$, are only the functions of $\omega$, respectively.

\subsection{Cracked Beam}

A beam with a crack can be regarded as two intact beam segments linked by the crack. Figure 1 shows a beam with a through-width single-sided crack specified by the dimensionless location $x=X / L$ and the dimensionless depth $y=d / H$, respectively. This crack connects two undamaged beam segments for each beam segment, the transverse vibration can be described in terms of Equations (2a) and (2b) as

$$
\begin{array}{ll}
0 \leq v \leq x: & W_{1}(v)=A_{1} \cosh \lambda_{1} v+A_{2} \sinh \lambda_{1} v+A_{3} \cos \lambda_{2} v+A_{4} \sin \lambda_{2} v \\
& \psi_{1}(v)=A_{2} m_{1} \cosh \lambda_{1} v+A_{1} m_{1} \sinh \lambda_{1} v-A_{4} m_{2} \cos \lambda_{2} v+A_{3} m_{2} \sin \lambda_{2} v \\
x \leq v \leq 1: & W_{2}(v)=B_{1} \cosh \lambda_{1} v+B_{2} \sinh \lambda_{1} v+B_{3} \cos \lambda_{2} v+B_{4} \sin \lambda_{2} v \\
& \psi_{2}(v)=B_{2} m_{1} \cosh \lambda_{1} v+B_{1} m_{1} \sinh \lambda_{1} v-B_{4} m_{2} \cos \lambda_{2} v+B_{3} m_{2} \sin \lambda_{2} v
\end{array}
$$

where $W_{1}(v)$ and $W_{2}(v)$ are the transverse deflections of two intact beam segments at the location $v$, respectively. $\Psi_{1}(v)$ and $\Psi_{2}(v)$ are the rotation angles of two intact beam segments at the location $v$, respectively. $A_{i}$ and $B_{i}(i=1-4)$ are constants associated with the boundary and continuity conditions at the crack location, which are as follows:

$$
\begin{gathered}
\left.W_{1}\right|_{v=0}=0,\left.\Psi_{1}\right|_{v=0}=0,\left.\Psi_{2}^{\prime}\right|_{v=1}=0,\left.\left(W_{2}^{\prime}-\Psi_{2}\right)\right|_{v=1}=0 \\
\left.W_{1}\right|_{v=x}=\left.\left.W_{2}\right|_{v=x}\left(\Psi_{2}-\Psi_{1}\right)\right|_{v=x}=\left.\theta \Psi_{2}^{\prime}\right|_{v=x},\left.\Psi_{1}^{\prime}\right|_{v=x}=\left.\Psi_{2}^{\prime}\right|_{v=x} \\
\left.\left(W_{1}^{\prime}-\Psi_{1}\right)\right|_{v=x}=\left.\left(W_{2}^{\prime}-\Psi_{2}\right)\right|_{v=x}
\end{gathered}
$$

where $\theta(y)$ is the dimensionless crack-sectional flexibility [42]:

$$
\theta(y)=6 \pi y^{2} f(y)(H / L)
$$

where $f(y)$ is a damage correction factor [43], which can be expressed as

$$
f(y)=\frac{0.923+0.199\left(1-\sin \left(\frac{\pi}{2} y\right)\right)^{4}}{\cos \left(\frac{\pi}{2} y\right)} \sqrt{\frac{\tan \left(\frac{\pi}{2} y\right)}{\frac{\pi}{2} y}}
$$

From Equations (4) to (6), the frequency characteristic equation depicting the transverse vibration of the cracked beam can be derived as [44]

$$
p\left(m_{1}, m_{2}, \lambda_{1}, \lambda_{2}, x\right) \theta(y)+q\left(m_{1}, m_{2}, \lambda_{1}, \lambda_{2}\right)=0
$$

in which $p$ and $q$ are given as 


$$
\begin{aligned}
& p\left(m_{1}, m_{2}, \lambda_{1}, \lambda_{2}, x\right) \\
& =\left[m_{2} \lambda_{2}\left(m_{1}-\lambda_{1}\right) \cosh (1-x) \lambda_{1} \sin (1-x) \lambda_{2}+m_{1} \lambda_{1}\left(m_{2}+\lambda_{2}\right) \sinh (1-x) \lambda_{1} \cos (1-x) \lambda_{2}\right] \times \\
& {\left[\left(m_{1} m_{2}^{2} \lambda_{2}+m_{2} m_{1}^{2} \lambda_{1}+m_{1}^{2} \lambda_{1} \lambda_{2}-m_{1}^{2} \lambda_{1} \lambda_{2}\right) \cosh \lambda_{1} x \cos \lambda_{2} x-\right.} \\
& m_{1} m_{2}\left(m_{2} \lambda_{1}-m_{1} \lambda_{2}+2 \lambda_{1} \lambda_{2}\right) \sinh \lambda_{1} x \sin \lambda_{2} x- \\
& \left.m_{1} m_{2}\left(\lambda_{2}^{2}-\lambda_{1}^{2}+m_{1} \lambda_{1}+m_{2} \lambda_{2}\right)-m_{1} \cosh \lambda_{1} x \sin \lambda_{2} x+m_{2} \sinh \lambda_{1} x \cos \lambda_{2} x\right] \times \\
& {\left[2 m_{1} m_{2} \lambda_{1} \lambda_{2}\left(m_{2}+\lambda_{2}\right)\left(m_{1}-\lambda_{1}\right)\left(1-\cosh (1-x) \lambda_{1} \cos (1-x) \lambda_{2}\right) \times\right.} \\
& \left.\left(m_{1}^{2} \lambda_{1}^{2}\left(m_{2}+\lambda_{2}\right)^{2}-m_{2}^{2} \lambda_{2}^{2}\left(m_{1}-\lambda_{1}\right)^{2}\right) \sinh (1-x) \lambda_{1} \sin (1-x) \lambda_{2}\right] \\
& q\left(m_{1}, m_{2}, \lambda_{1}, \lambda_{2}\right) \\
& =\left(m_{1} \lambda_{2}+m_{2} \lambda_{1}\right)\left[m_{2}^{2} \lambda_{2}\left(m_{1}-\lambda_{1}\right)+m_{1}^{2} \lambda_{1}\left(m_{2}+\lambda_{2}\right) \cos \lambda_{1} \cos h \lambda_{1}+\right. \\
& \left.m_{1} m_{2}\left(m_{2} \lambda_{1}-m_{1} \lambda_{2}+2 \lambda_{1} \lambda_{2}\right) \sinh \lambda_{1} \sin \lambda_{2}-m_{1} m_{2}\left(\lambda_{2}^{2}-\lambda_{1}^{2}+m_{1} \lambda_{1}+m_{2} \lambda_{2}\right)\right]
\end{aligned}
$$

Equation (8) presents an implicit function of the eigen-frequency, $\omega$, the dimensionless crack parameters, $x$ and $y$. When the beam is certain, its geometric and physical parameter will be certain. The unknown parameters are only $\omega, x$ and $y$. Therefore, the relationship of $\omega, x$ and $y$ can be presented in a three-dimensional coordinate system. Moreover, when the crack is known, the eigen-frequency can be solved, whereby the transverse vibration of the beam can be obtained.

\section{Existing Frequency Contour-Line Method with Limitations in Portraying Damage 3.1. Eigen-Frequency Panorama}

For a crack in a beam, the possible variation of crack depth at a crack location that varies throughout the beam length can evoke an overall set of crack scenarios. This overall set collectively characterizes all crack situations of the beam, which can be analytically depicted by the solutions of Equation (8) with crack location and severity as independent variables and eigen-frequency as dependent variable. In theory, for a given crack scenario of a continuous beam with specific $x$ and $y$, Equation (8) can produce a series of eigenfrequencies from the 1st order to the infinite order due to the infinite degrees of freedom (DOFs) for a continuous beam. All eigen-frequencies arising from Equation (8) can constitute an eigen-frequency panorama that comprehensively reflects the effect of all possible crack scenarios on the dynamic property of the beam in the situation of unlimited computer resource. In reality, the finite instead of infinite DOFs are usually considered for a beam, leading to finite eigen-frequencies. To this end, the eigen-frequency panorama for a cracked beam involves the first $k$ eigen-frequencies out of the overall set of crack scenarios. Figure 2 illustrates the eigen-frequency panorama involving the first three eigen-frequencies for a certain cantilever beam bearing a crack. The eigen-frequency panorama comprises three eigen-frequency surfaces labeled by $\omega_{1}, \omega_{2}, \omega_{3}$, each consisting of eigen-frequencies of the same order as functions of crack locations and severities. This eigen-frequency panorama reflects the effect of all possible cracks in the beam on its first three eigen-frequencies.

\subsection{Frequency Contour-Line Method for Crack Identification}

From the perspective of forward problem, an eigen-frequency panorama comprehensively depicts the effect of all possible crack scenarios on the dynamic property of a beam. For an arbitrary crack at the point $(x, y)$, the associated eigen-frequencies can be specified from the eigen-frequency panorama arising from Equation (8). 


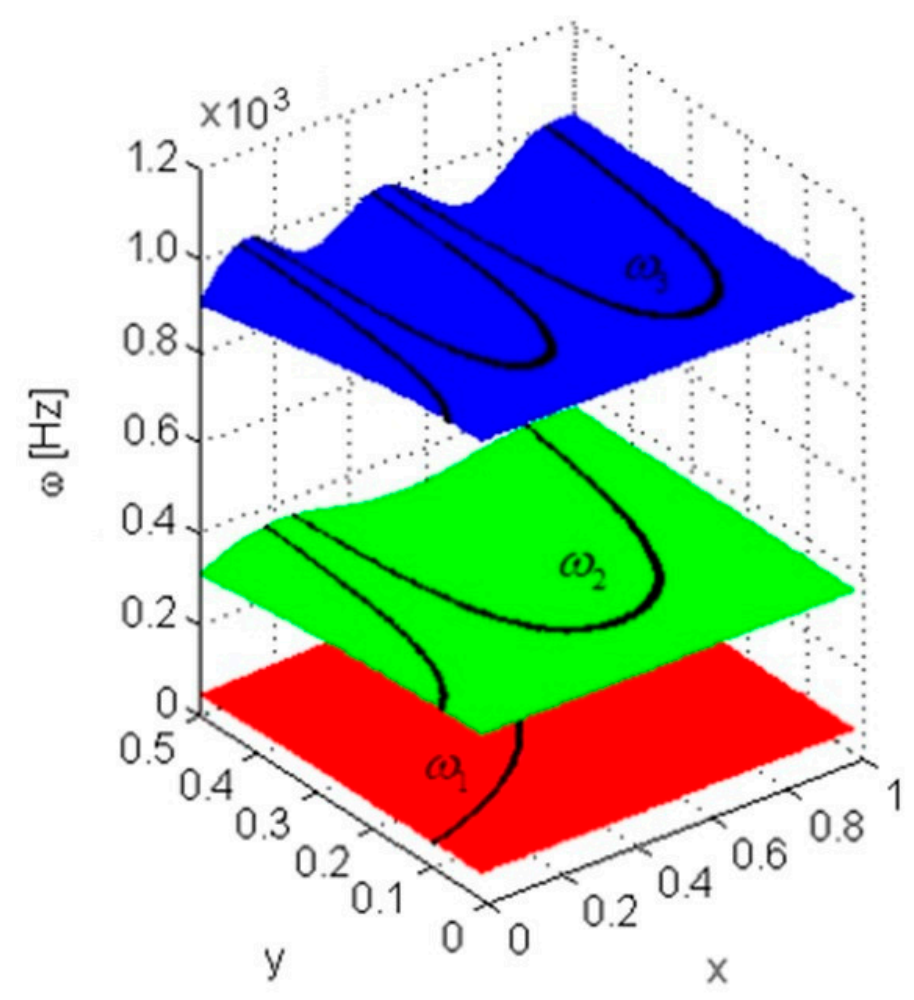

Figure 2. The eigen-frequency panorama comprising the first three eigen-frequencies for a certain cantilever beam bearing a crack.

From the perspective of an inverse problem, an eigen-frequency panorama along with experimentally obtained eigen-frequencies provides a strategy of identifying a crack in a beam. Consider a beam of the prior knowledge: bearing an unknown crack and experimentally acquired eigen-frequencies, $\omega_{i}, \mathrm{i}=1,2, \cdots, n$, with the question to identify the crack. One can obtain the eigen-frequency panorama of the beam in terms of Equation (8a), and $\omega_{i}, \mathrm{i}=1,2, \cdots, n$ are discrete points of the eigen-frequency panorama in the $\omega$ axis. Thus, $\omega_{i}$ can provoke a horizontal plane cutting through the $\omega_{i}$-labeled eigen-frequency surface of the eigen-frequency panorama, leading to a frequency contour line. All the points of this frequency contour line designate all possible crack scenarios specified by $\omega_{i}$. The frequency contour line is further projected onto the $x-y$ plane, giving the frequency projection curve indicating the possible locations and depths of the crack. In general, for $\omega_{i}, \mathrm{i}=1,2, \cdots, n$, all the frequency projection curves can be produced from frequency contour lines. The common intersection points of all the frequency projection curves designate the location and depth of the crack under inspection. Such a crack identification method is termed frequency contour-line method that uses measured eigen-frequencies with eigen-frequency panorama to identify a crack in a beam.

The procedure of identifying a crack using the frequency contour-line method is illustrated on a beam with a cantilever crack. (i) Eigen-frequency panorama. Figure 3 presents the first three eigen-frequencies-led eigen-frequency panorama, generated by Equation (8). (ii) Experimental eigen-frequencies. Three eigen-frequencies, $\omega_{1}, \omega_{2}, \omega_{3}$, are acquired through experimental measurements. (iii) Frequency contour lines. $\omega_{1}, \omega_{2}, \omega_{3}$ provoke three frequency contour lines laying on the eigen-frequency panorama, as shown in Figure 3. The extracted frequency contour lines are also presented in Figure 4. (iv) Frequency projection lines. Frequency contour lines in Figure 4 are projected on the $x-y$ plane, leading to the frequency projection lines as shown in Figure 5a, in which a common intersection point $\mathrm{O}$ can be observed. (iv) Crack parameter. The point $\mathrm{O}$, as zoomed-in in Figure $5 b$, designates the dimensionless crack location $x$ and the dimensionless depth $y$ of the crack under investigation. 


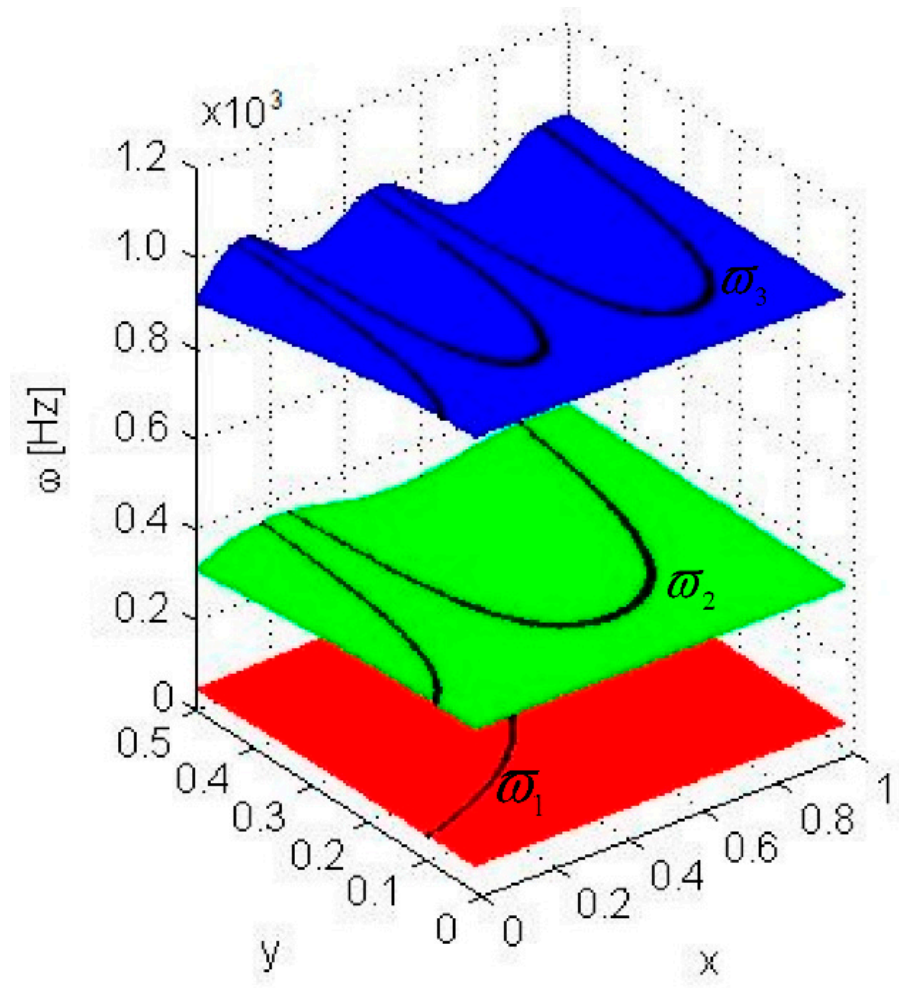

Figure 3. Eigen-frequency panorama with frequency contour lines. $\omega_{1}, \omega_{2}, \omega_{3}$.

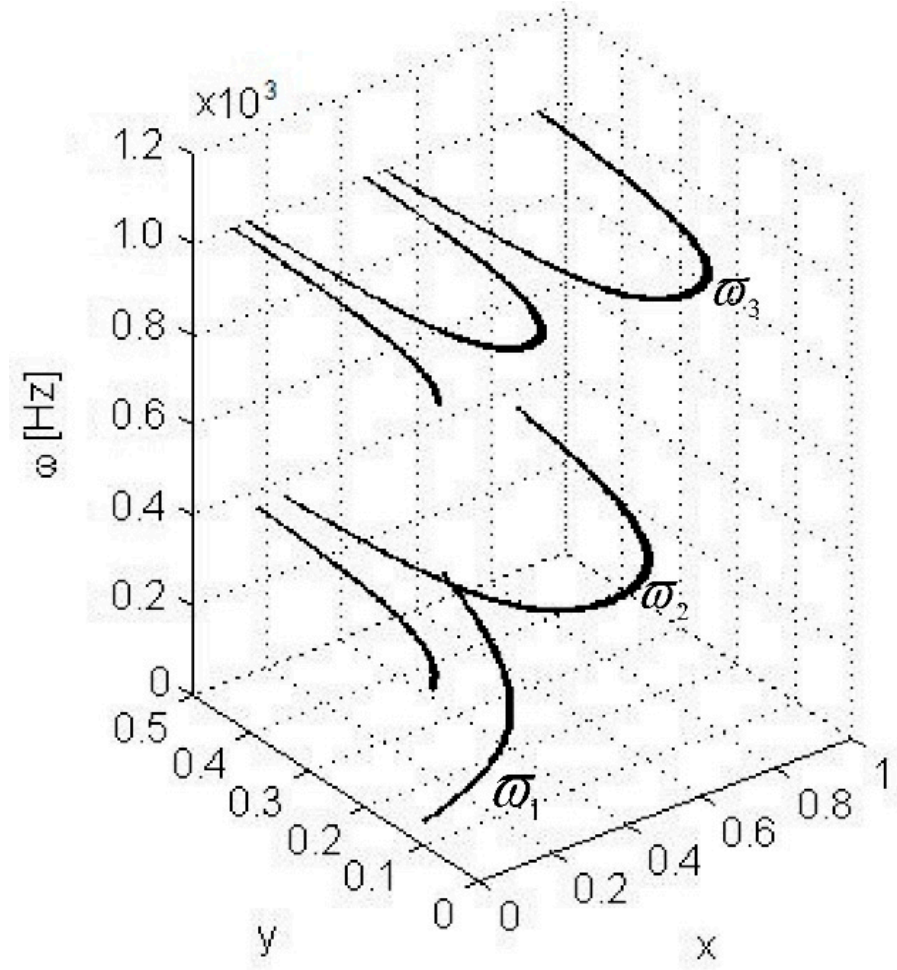

Figure 4. Extracted frequency contour lines. 


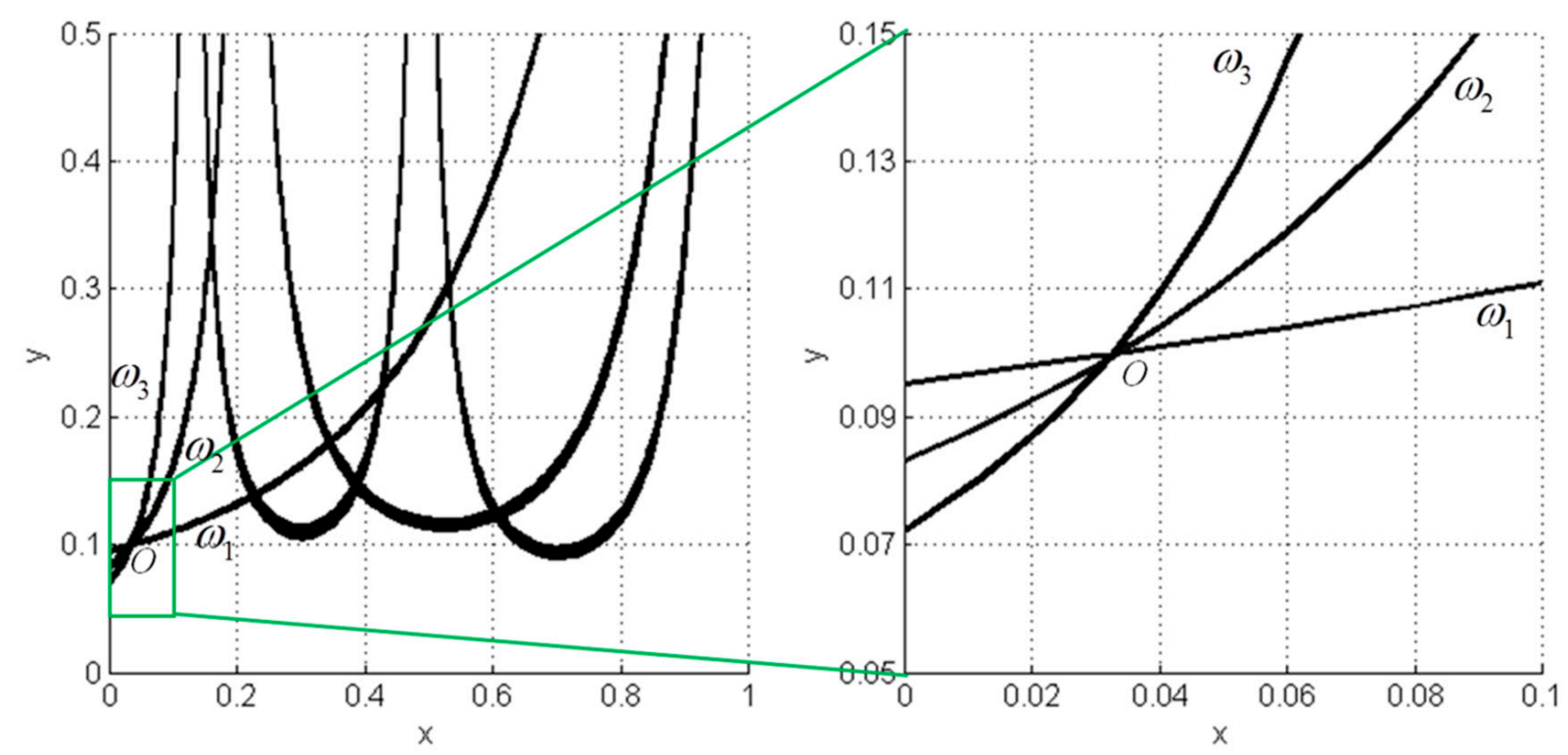

(a)

(b)

Figure 5. Frequency projection lines. (a) projection lines with common intersection point O. (b) Zoomed-in point $\mathrm{O}$ indicating crack parameter.

\subsection{Limitations in Frequency Contour-Line Method}

Despite the remarkable characteristic, the frequency contour-line method has substantial limitations in identification of a crack in a beam. The primary two limitations are clarified in the following.

\subsubsection{Inefficiency in Establishing Eigen-Frequency Panorama}

Establishment of the eigen-frequency panorama is the basic component of identifying a crack using the frequency contour-line method, where the panorama implies that numerous eigen-frequencies need to be evaluated. Furthermore, one eigen-frequency of an eigen-frequency panorama is obtained by solving the frequency characteristic equation (Equation (8)). Notably, Equation (8a) involves complex transcendental functions with sinhand cosh-functions as essential elements, incurring extreme nonlinearity and complexity. In the frequency contour-line method, the frequency characteristic equation is solved by numerical methods, typically the direct determinant evaluation (DDE) method. The key of the DDE is to evaluate the value of every independent element of the frequency determinant at a given value of $\omega$. Accordingly, the frequency determinant is calculated by pure algebraic operations of the determination expansion. The solutions of eigen-frequencies could be determined from the zero-crossings of the profile of frequency determinant versus $\omega$. It is obvious that it needs large amount of computation to produce one eigen-frequency value. To establish the eigen-frequency panorama, a large number of eigen-frequencies should be evaluated, resulting in huge computation cost and intractable manipulation. As a result, the inefficiency in establishing the eigen-frequency panorama largely influences the use of the frequency contour-line method to identify cracks in beams.

\subsubsection{Incompetence to Identify Cracks in Noisy Conditions}

In terms of the contour-line method, when experimentally obtained eigen-frequencies, $\omega_{i}, \mathrm{i}=1,2, \cdots, n$, are exactly the points of the eigen-frequency panorama of the beam, the intersection point of projection curves, stemming from intersection contour lines between the eigen-frequency panorama and virtual horizontal planes incurred by $\omega_{i}, \mathrm{i}=1,2, \cdots, n$, can exactly point out the location and depth of the crack. Such a procedure is illustrated in Figures $2-5$, where we assume that the experimentally obtained three eigen-frequencies, 
$\omega_{i}, \mathrm{i}=1,2, \cdots, n$ are exactly the points of the eigen-frequency panorama, the intersection point $\mathrm{O}$ designates the genuine crack. Experimental acquisition of eigen-frequencies, $\omega_{i}, \mathrm{i}=1,2, \cdots, n$, is another key component for crack identification relying on the frequency contour-line method. During the course of actual testing, random noise is inevitably involved in the acquired eigen-frequencies, leading to $\omega_{i}^{*}, \mathrm{i}=1,2, \cdots, n$, which are deviated from the exact natural frequencies $\omega_{i}, \mathrm{i}=1,2, \cdots, n$. As performing the frequency contour-line method to identify a crack, such deviations will further pass down into the frequency contour lines and, further, the frequency projection lines in the $x-y$ plane. Finally, the frequency projection lines do not intersect at a common point, as illustrated in Figure 6, where the noise-incurred deviation of $\omega_{i}^{*}, \mathrm{i}=1,2, \cdots, n$ from $\omega_{i}, \mathrm{i}=1,2, \cdots, n$ is $0.1 \%$ in the magnitude of the latter. In such a situation, Nandwana and Maiti [45] prosed a method to estimate the crack location and depth from a chosen intersectional triangle of projection curves. Nevertheless, different results of crack identification can be realized for a single crack when considering the noise randomness. Therefore, the frequency contour-line method cannot judicially reflect a crack under noisy conditions.

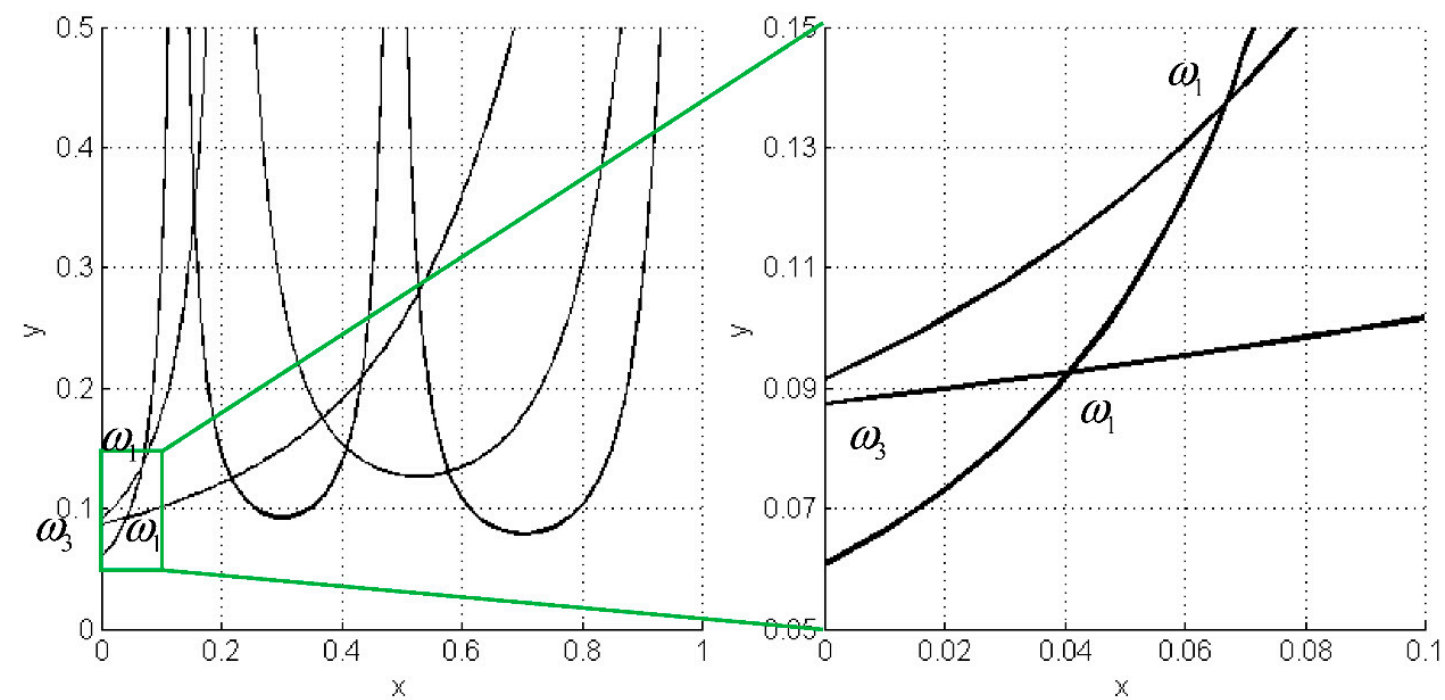

Figure 6. Illustration on failure of the frequency contour-line method in identifying a crack under noise conditions.

\section{Proposed Frequency Contour-Strip Method with Superiority in Characterizing Damage}

A frequency contour-strip method, acting as an upgradation of the frequency contourline method, is formulated by addressing the aforementioned limitations of inefficiency in establishing the eigen-frequency panorama and incompetence to identify a crack in noisy conditions in the latter method.

\subsection{Instant Establishment of Eigen-Frequency Panorama}

In the frequency contour-line method, the establishment of eigen-frequency panorama needs repetitive manipulations of numerically solving Equation (8) to produce eigenfrequency values point by point, incurring huge cost and computation time. To overcome such a deficiency, the symbolic operation of solving implicit functions is studied to tackle Equation (8), since it is an implicit function of $\omega, x$, and $y$. The symbolic operation is implemented in Matlab using the function isosurface that has the advanced mechanism of evaluating and visualizing implicit functions in an instant mode. Herein, the function isosurface is adopted to instantly solve Equation (8) by conquering the limitation of inefficiency to establish the eigen-frequency panorama in the frequency contour-line method. The procedure of using the function isosurface to evaluate and visualize Equation (8) to produce the eigen-frequency panorama is tabulated in Table 1, where crl_x, crd_y, feq_ $\omega$ denote the variable domains of crack location, crack depth and eigen-frequency, respectively. 
Table 1. Procedure of generating eigen-frequency panorama of a cracked beam.

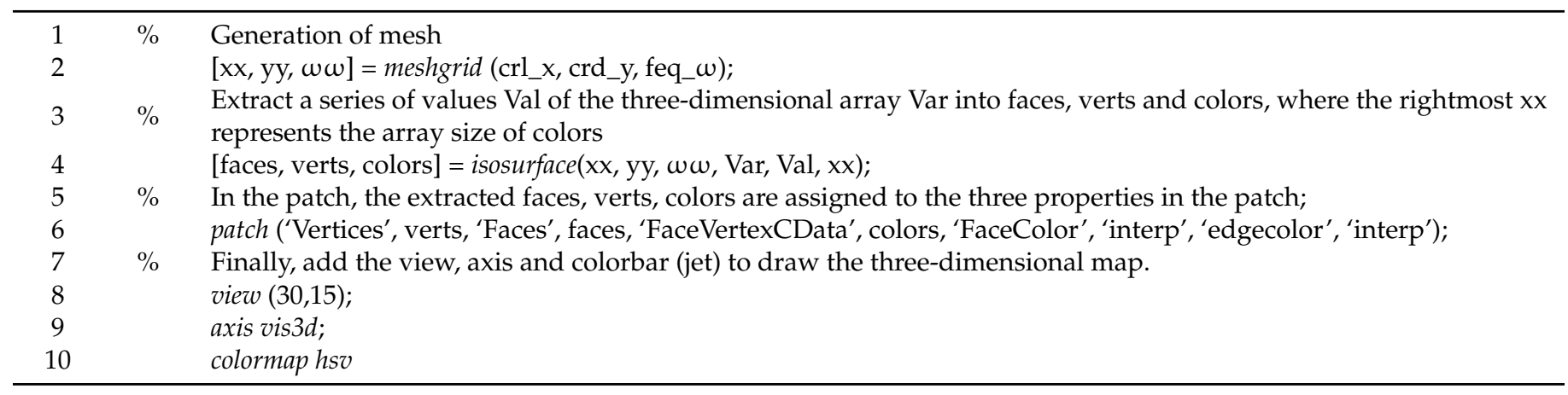

The function isosurface is a highly integrated module featuring smooth symbol operation, totally different from the DDE method and other numerical methods used in the contour-line method. With this merit, the function isosurface can produce an eigenfrequency panorama with a computational efficiency several orders of magnitude higher than general numerical methods in the frequency contour-line method.

A steel cantilevered cracked beam with length $500 \mathrm{~mm}$, thickness $19 \mathrm{~mm}$, and width $12 \mathrm{~mm}$ is used to illustrate the use of the function isosurface to produce the eigen-frequency panorama. For the beam, the elastic modulus, shear modulus, Poisson's ratio $(v)$, shear coefficient, and density are $184 \mathrm{GPa}, 86 \mathrm{GPa}, 0.3,10(1+v) /(12+11 v)$, and $7758 \mathrm{~kg} / \mathrm{m}^{3}$, respectively. With these parameters, Equation (8) provides a determined implicit function of $\omega, \mathrm{x}$, and $\mathrm{y}$. The function isosurface is used to solve this implicit function. The resulting eigen-frequency panorama involving the first 15 eigen-frequencies is shown in Figure $7 \mathrm{a}$. To demonstrate the generality, the eigen-frequency panorama of a simply-supported beam with the same materials and the geometrical parameters is produced using the function isosurface, as shown in Figure 7b. The total computational duration using an in-home laptop is about $12 \mathrm{~s}$ for the above two cases, whereas the DDE method in the frequency contourline method needs two and half hours to generate the eigen-frequency panoramas, with some numerical computation problems when the computation order of eigen-frequencies is higher than 12. To present the details of the eigen-frequency panorama, Figure 8 presents the first six order extracted eigen-frequency panoramas where the effect of a crack on each eigen-frequency is clear. As a result, the function isosurface provides a means of instantly establishing the eigen-frequency panorama, overcoming the inefficiency in obtaining the eigen-frequency panorama through finite element modeling or experiments.

\section{2. $\varepsilon$-Noise Cube}

The frequency contour-line method lacks a mechanism of reflecting the effect of random noise involved in measured eigen-frequencies on crack identification, giving rise to a specific estimate of possible crack scenarios. To investigate the effect of random noise on crack identification, a $\varepsilon$-noise cube is elaborated to depict the first three measured eigen-frequencies, shown as Figure 9. The $\varepsilon$-noise cube is defined by

$$
\omega_{1}^{*}=\omega_{1}(1+i \cdot \varepsilon), \omega_{2}^{*}=\omega_{2}(1+j \cdot \varepsilon), \omega_{3}^{*}=\omega_{3}(1+j \cdot \varepsilon)
$$

where $\omega_{l}, l=1-3$, are the exact first three eigen-frequencies, and $\omega_{m}^{*}, m=1-3$, are boundary values of measured eigen-frequencies under the random noise level $\varepsilon$. According to the $\varepsilon$-noise cube, there could be eight directional deviations of $\omega_{m}^{*}$ from $\omega_{l}$, specified by the vertices of the cube. Consequently, eight groups of $\omega_{m}^{*}, m=1-3$, are required in light of the $\varepsilon$-noise cube to bound the possible variations of measured eigen-frequencies for each crack case. Without loss of generality, a $\varepsilon$-noise hypercube can be created when the first $n$ measured eigen-frequencies are employed to identify a crack. 


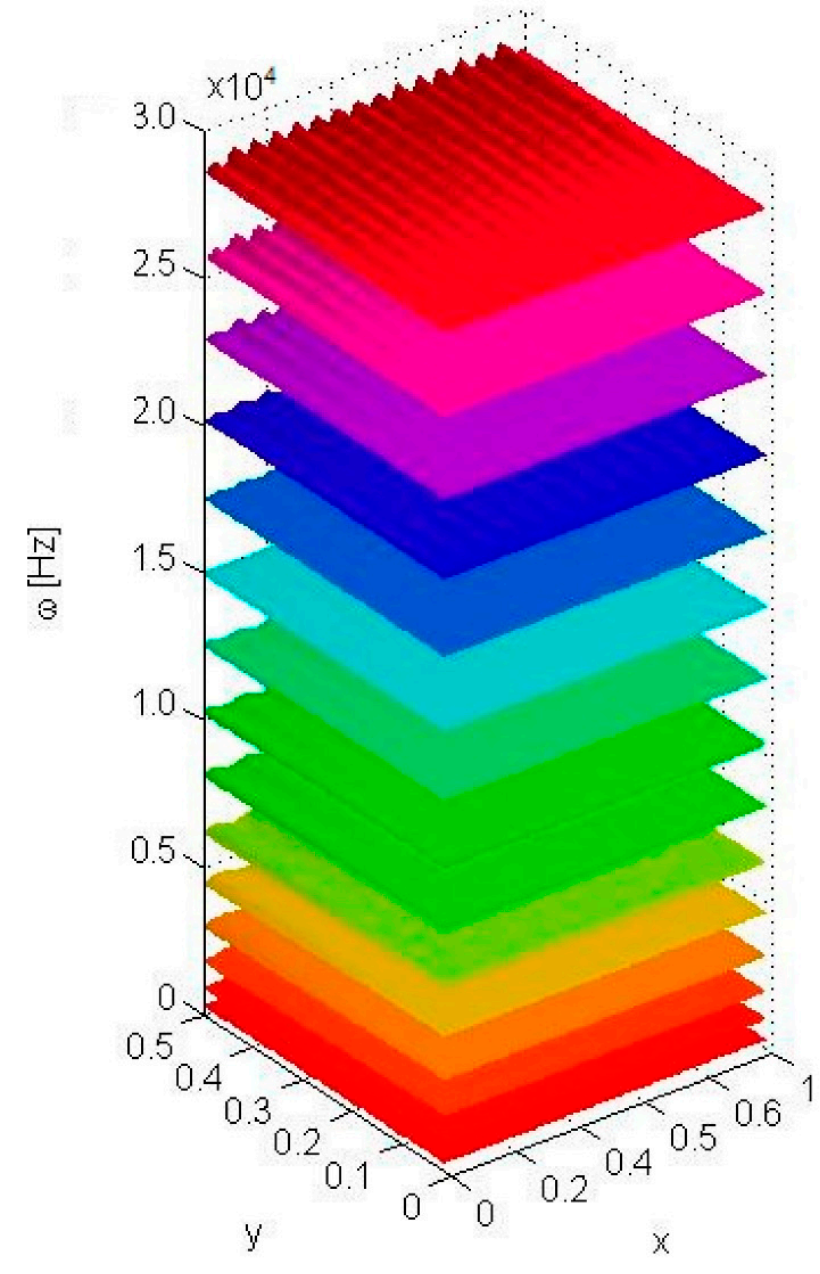

(a)

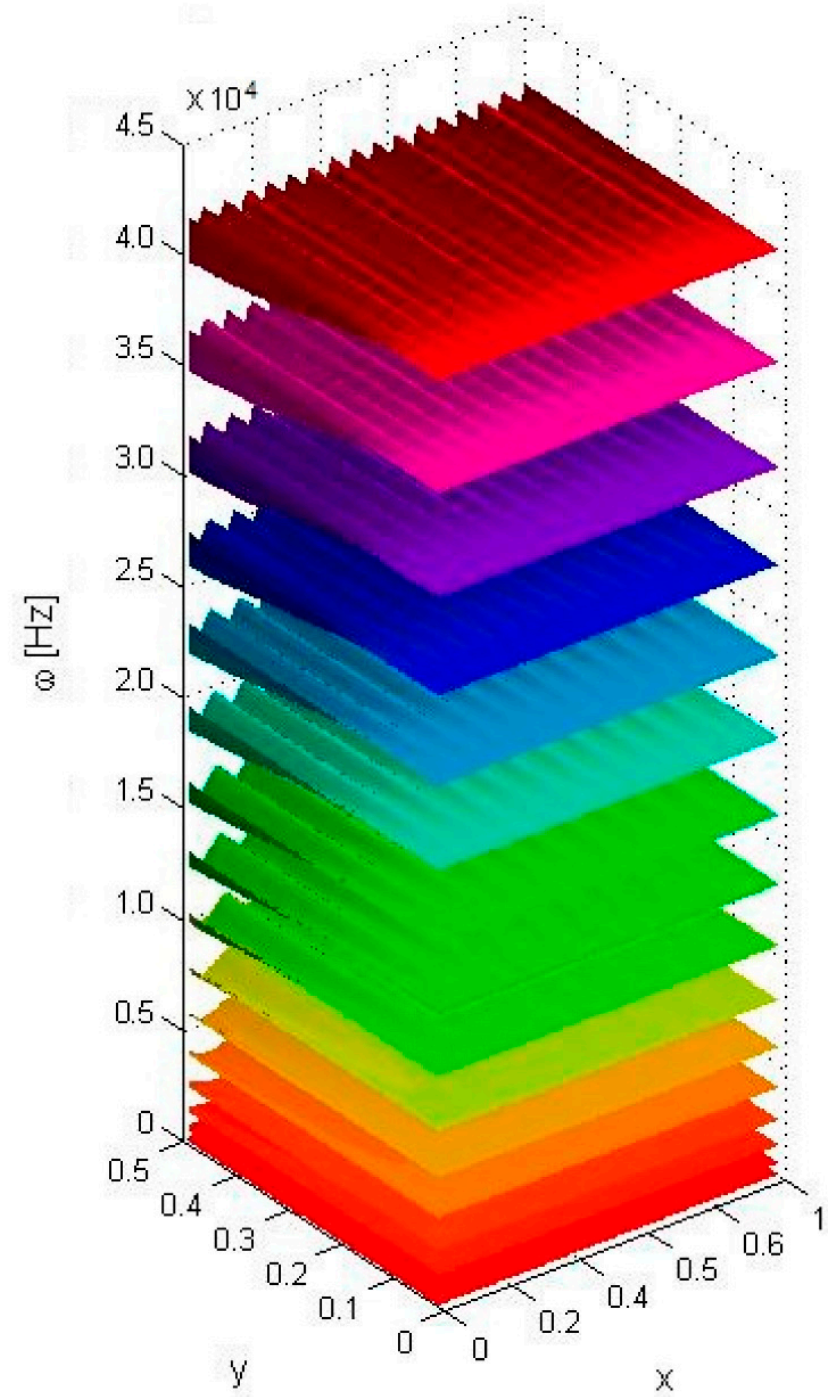

(b)

Figure 7. Eigen-frequency panoramas generated using the function isosurface. (a) Cantilever beam; (b) simply-supported beam.

Considering the randomness of noise in measurements, the measured eigen-frequencies, i.e., $\left(\omega_{1}^{*}, \omega_{2}^{*}, \omega_{3}^{*}\right)$, can be expressed in terms of the $\varepsilon$-noise cube as

$$
\begin{aligned}
& \omega_{1}\left(1-\varepsilon_{1}\right) \leq \omega_{1}^{*} \leq \omega_{1}\left(1+\varepsilon_{1}\right) \\
& \omega_{2}\left(1-\varepsilon_{2}\right) \leq \omega_{2}^{*} \leq \omega_{2}\left(1+\varepsilon_{2}\right) \\
& \omega_{3}\left(1-\varepsilon_{3}\right) \leq \omega_{3}^{*} \leq \omega_{3}\left(1+\varepsilon_{3}\right)
\end{aligned}
$$

In practical crack identification, $\omega_{1}, \omega_{2}, \omega_{3}$ and $\varepsilon$ are determined by the mean and the standard variance of a large number of measured eigen-frequencies. 

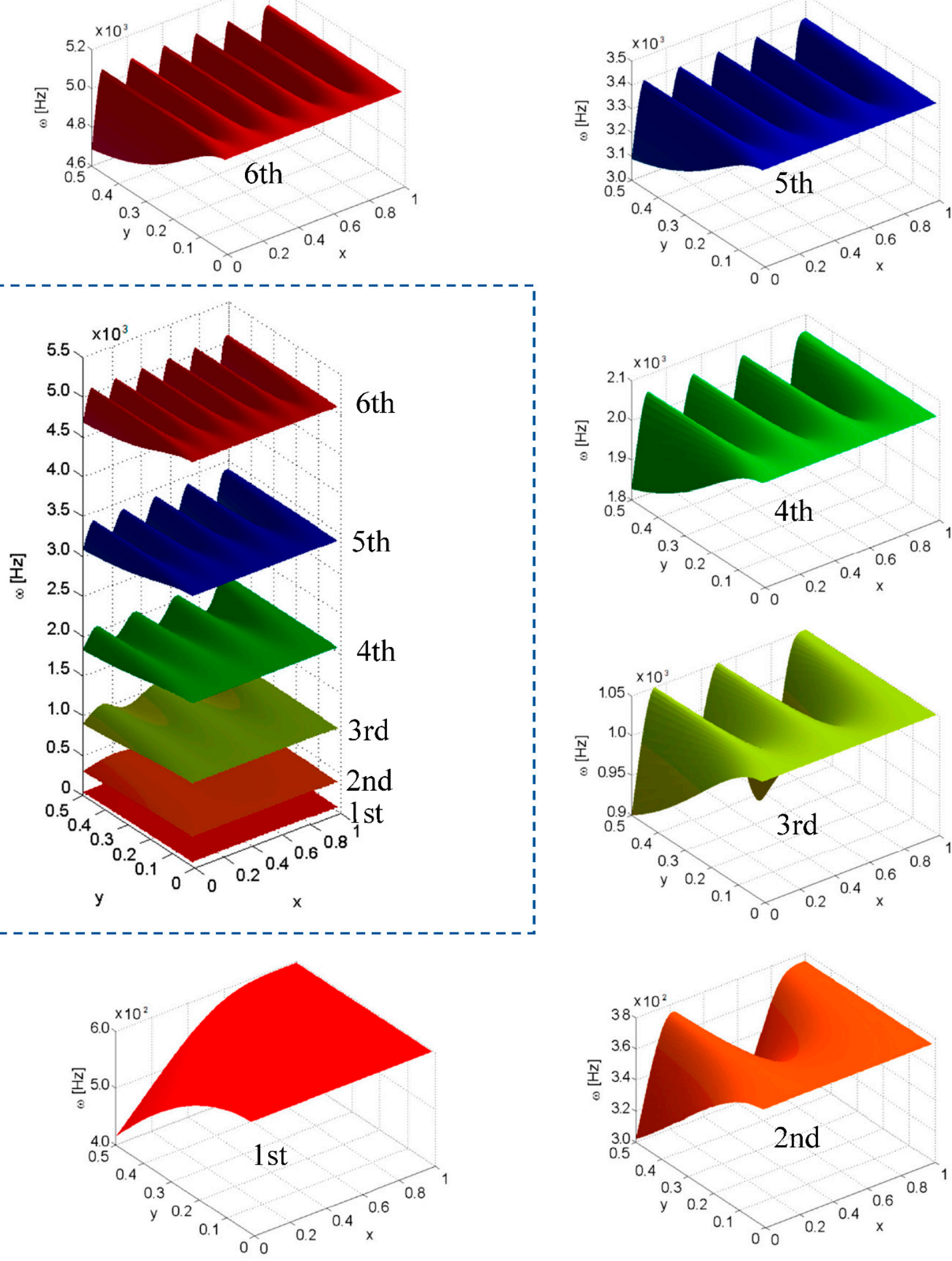

Figure 8. Detailed illustration on eigen-frequency panorama generated by the function isosurface. 


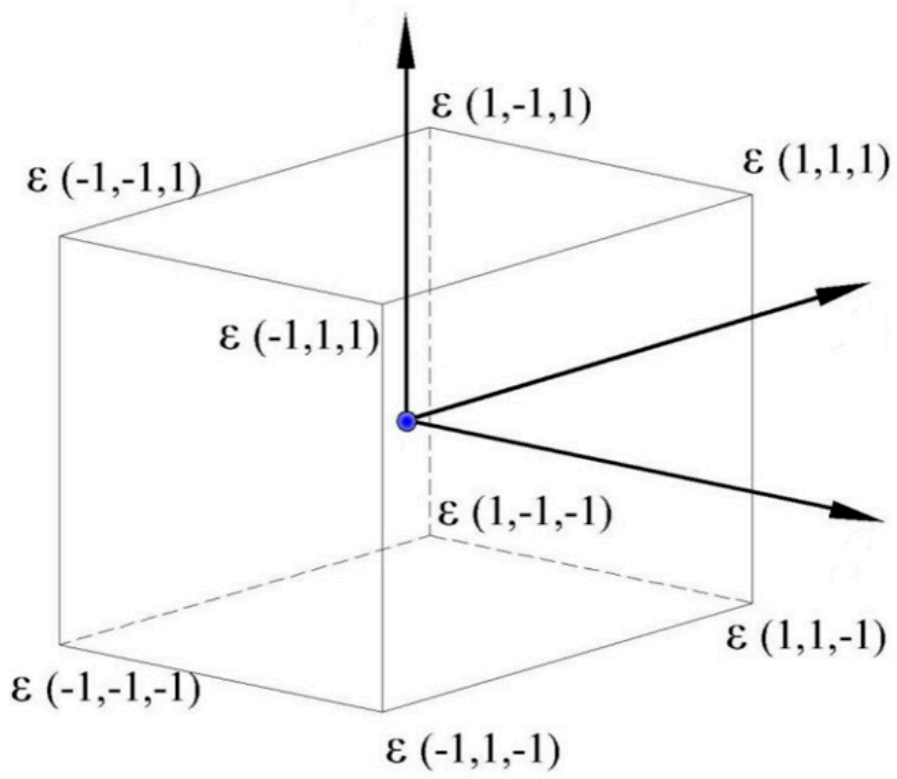

Figure 9. $\varepsilon$-noise cube.

\subsection{Contour-Strip Method Fourmulation}

Considering the randomness of noise, the measured $i$ th eigen-frequency, $\omega_{i}^{*}$, is really a variable occupying an interval $\omega_{i}\left(1-\varepsilon_{i}\right)<\omega_{i}^{*}<\omega_{i}\left(1+\varepsilon_{i}\right)$ in the light of Equation (10). In the eigen-frequency panorama, $\omega_{i}\left(1-\varepsilon_{i}\right)$ and $\omega_{i}\left(1+\varepsilon_{i}\right)$ can evoke two frequency contour-lines between which the bounded area forms a strip. Owing to the usual small value of $\varepsilon_{i}$, this strip is relatively narrow, roughly giving rise to a frequency contour-strip of the eigen-frequency panorama. The projection of the frequency contour-strip onto the $x-y$ plane produces a frequency projection strip that contains possible estimates of the crack in terms of the $i$ th eigen-frequency under noise conditions. In a similar way, when $\omega_{i}^{*}, i=1,2, \cdots, n$, is considered, $n$ frequency projection strips can be obtained in the $x-y$ plane, each giving possible estimates of the crack with respect to that eigen-frequency. The intersection region of $n$ frequency projection strips specify the possible locations and depths of the crack. This procedure constitutes the frequency contour-strip method for crack identification of a beam with a crack, which is a promotion of the frequency contourline method, featuring accommodation of random noise to characterize damage in a beam. Figures 10 and 11 illustrate the use of the frequency contour-strip method involving the first three eigen-frequencies to identify a crack in the beam. According to the frequency contourstrip method, the resulting frequency contour-strips of the eigen-frequency panorama are shown in Figure 10a, while Figure 10b presents the extracted frequency contour-strips to provide a clearer presentation. Figure 11a shows the frequency projection strips, on which the zoomed-in intersection region $R$ is shown in Figure 11b, where the translucent setting of frequency projection strips clearly depicts $R$ as a polygon with $p$ vertexes $\left(x_{1}, y_{1}\right),\left(x_{2}, y_{2}\right)$, $\ldots,\left(x_{p}, y_{p}\right)$. Differing from the frequency contour-line method, which designates a crack with a single solution, the frequency contour-strip method characterizes a crack with a set of possible estimates of crack parameters. From the intersection region $R$, a sole estimate of crack location and depth, more suitable for characterizing a crack in noisy conditions, can be identified with $p$ vertices as

$$
\begin{aligned}
& \mu_{x}=E X=\frac{1}{p} \sum_{i=1}^{p} x_{i} \\
& \mu_{y}=E Y=\frac{1}{p} \sum_{i=1}^{p} y_{i}
\end{aligned}
$$




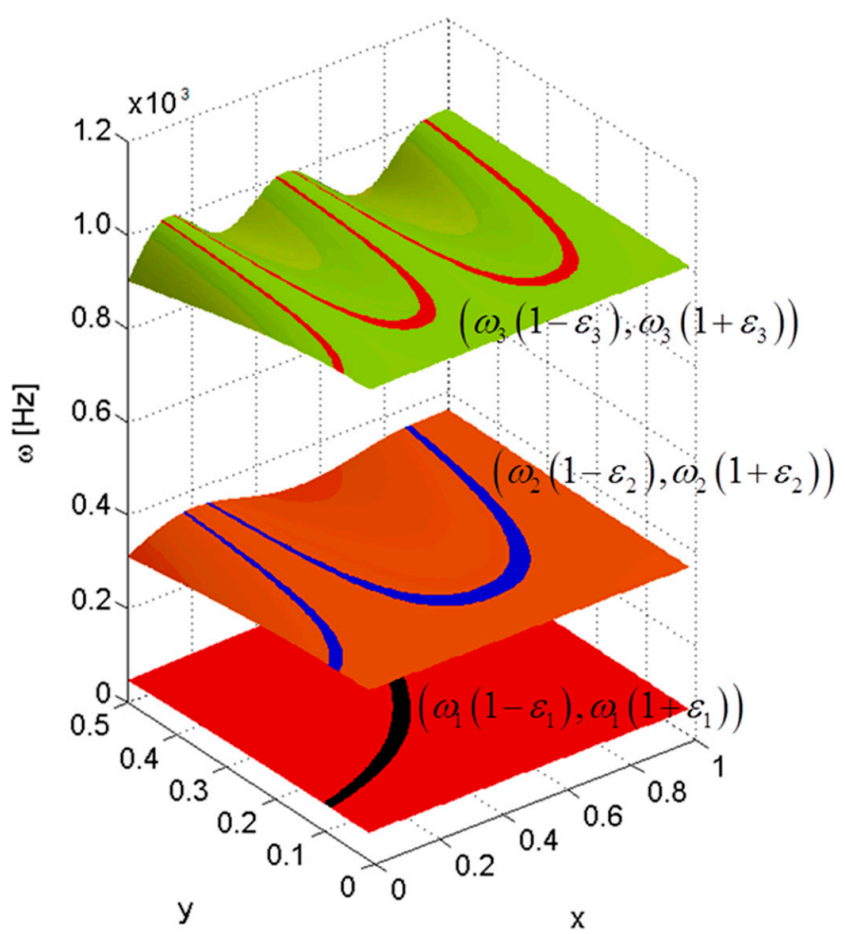

(a)

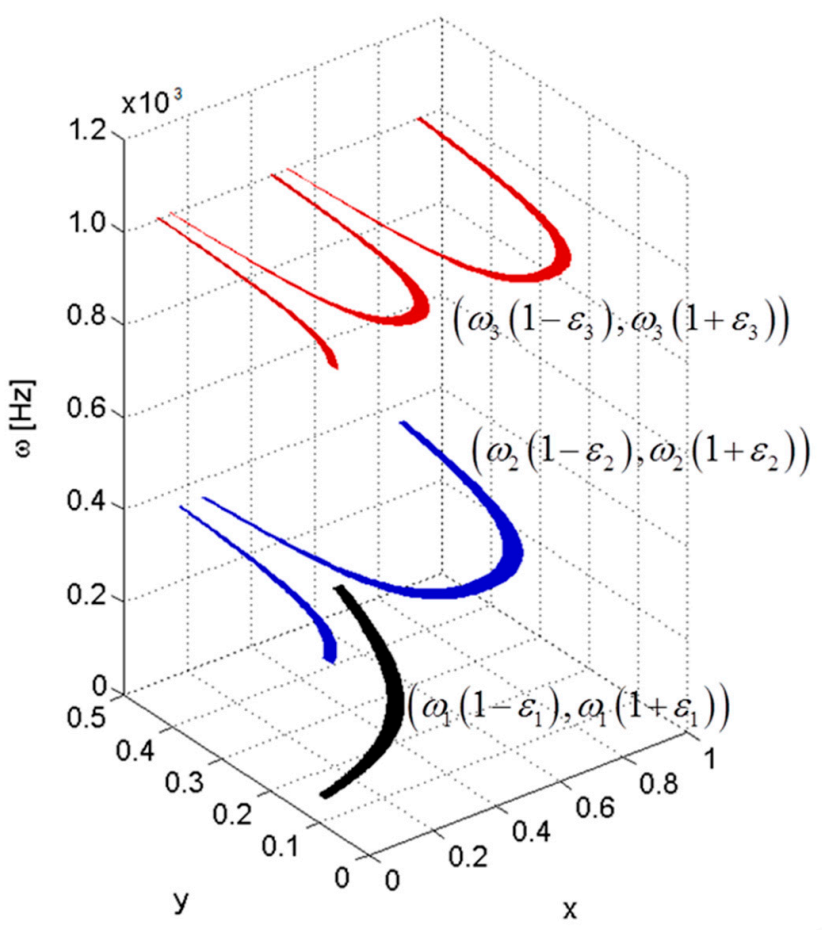

(b)

Figure 10. Frequency contour-strips. (a)Frequency contour-strips of the eigen-frequency panorama. (b) Extracted frequency contour-strips.

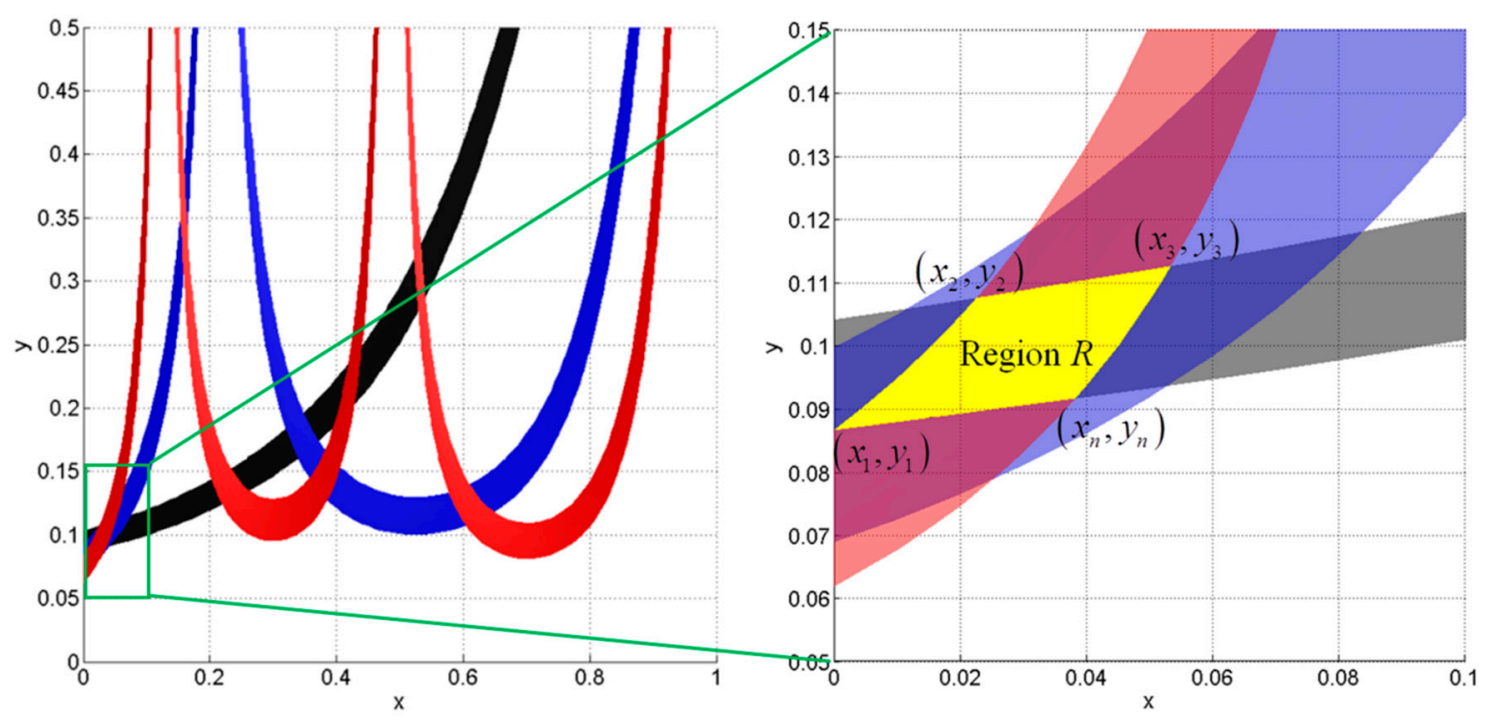

(a)

(b)

Figure 11. Frequency contour-strip. (a) Frequency projection strips. (b) Zoomed-in intersection region R.

\section{Experimental Validation}

\subsection{Experimental Description}

Experimental samples are fabricated using a laser-cutting machine to dissect a steel plate into beam segments. The specification parameters for the material and geometry of the steel plate are shown in Table 2. The length of each beam segment is $600 \mathrm{~mm}$, and the clamped part covers $100 \mathrm{~mm}$, leading to a cantilever beam of span $500 \mathrm{~mm}$. A through-width crack is introduced using a computer-controlled wire-cutting machine 
to cut the beam along one side. The wire of the machine has a diameter of $0.16 \mathrm{~mm}$, and resulting crack has a width between 0.16 and $0.18 \mathrm{~mm}$. For an arbitrarily chosen sample, Figure 12 presents the setup of the vibration-table dynamic testing. When a hammer is used to impact the beam, the vibration acceleration of the beam is recorded at the sampling frequency $12,800 \mathrm{~Hz}$ by an accelerometer mounted at the middle of the beam span. The acquired acceleration responses are preprocessed by rbio4. 4 wavelet filter for noise reduction. The type of accelerometer is 1A102E, the type of impact hammer is Modally Tuned (U.S. PAT. NO. 4.799.375), and the data acquisition facility is DH8303N with dynamic signal acquisition and analysis system. These devices are from Jiangsu Donghua Testing Technology Co., Ltd. Figure 13 illustrates the frequency spectra of the beam with a crack of the depth, $y$, increasing from $10 \%$ to $40 \%$ at the step of $10 \%$ at the location of $x=3.3 \%$.

Table 2. Specification parameters of material and geometry of steel plate.

\begin{tabular}{cccccccc}
\hline $\begin{array}{c}\text { Length } \\
(\mathbf{m m})\end{array}$ & $\begin{array}{c}\text { Thickness } \\
(\mathbf{m m})\end{array}$ & $\begin{array}{c}\text { Width } \\
(\mathbf{m m})\end{array}$ & $\begin{array}{c}\text { Density } \\
\left(\mathbf{k g} / \mathbf{m}^{3}\right)\end{array}$ & $\begin{array}{c}E \\
(\mathbf{G P a})\end{array}$ & $\begin{array}{c}G \\
(\mathbf{G P a})\end{array}$ & $\boldsymbol{v}$ & $\boldsymbol{k}$ \\
\hline 500 & $19 \mathrm{~mm}$ & $12 \mathrm{~mm}$ & 7758 & 184 & 86 & 0.3 & $10(1+v) /(12+11 v)$ \\
\hline
\end{tabular}

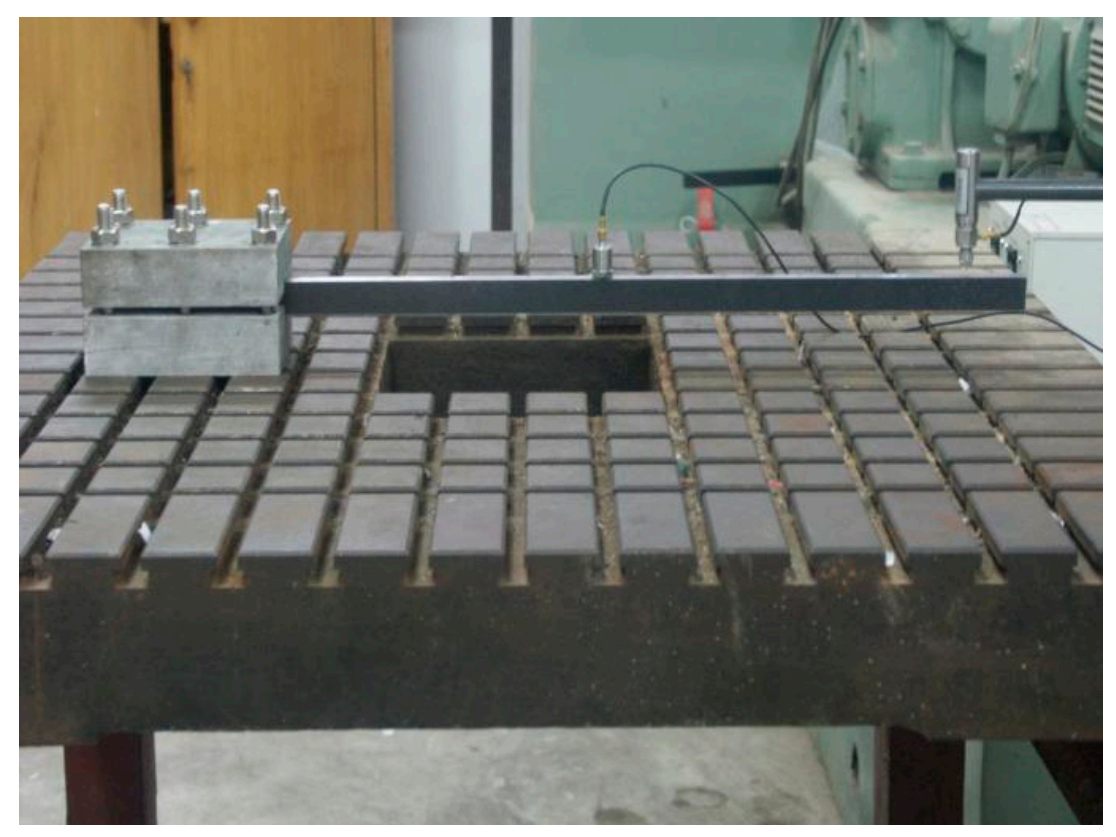

Figure 12. Setup of dynamic testing.

\subsection{Crack Identification Using Freqeuncy Contour-Strip Method}

Three crack cases shown in Table 3 are arbitrarily chosen to demonstrate the effectiveness of the frequency contour-strip method in damage identification.

\subsubsection{Case 1}

Case 1 is related to a crack, $x=3.3 \%$ and $y=10 \%$ in the cantilever beam (Figure 11b). Equation (8) with the parameters specified with the specification values in Table 2 forms the analytical model for characterizing the transverse vibration of the cracked cantilever beam. A comprehensive model calibration procedure is carried out for the experimentally measured eigen-frequencies considering all possible crack cases (not only Case 1), giving valid parameter values instead of specification parameter values to form an updated analytical model.

The function isosurface is used to solve the analytical model to produce the eigenfrequency panorama (Figures 7 and 8). Considering noise effect on eigen-frequencies, multiple tests are conducted to acquire a set of first three eigen-frequencies. The averages 
of this set of first 3 eigen-frequencies are $59.38 \mathrm{~Hz}, 372.27 \mathrm{~Hz}$, and $1043.55 \mathrm{~Hz}$, with standard variances 1.1, 1.8, and 3.7 that denote the noise effect. In terms of Equation (10), the frequency contour strips can be obtained as shown in Figures 10 and 11. From the frequency contour strips, the resulting frequency projection strips on the $x-y$ plane are presented in Figure 11, in which the intersection region $R$ of frequency projection strips can be observed in Figure 11b. According to Equation (11), the dimensionless crack parameters, $x$ and $y$ can be estimated from the intersection region $\mathrm{R}$ as $\mu_{x}=2.95 \%$ and $\mu_{y}=9.75 \%$, close to the genuine location and severity of the crack.

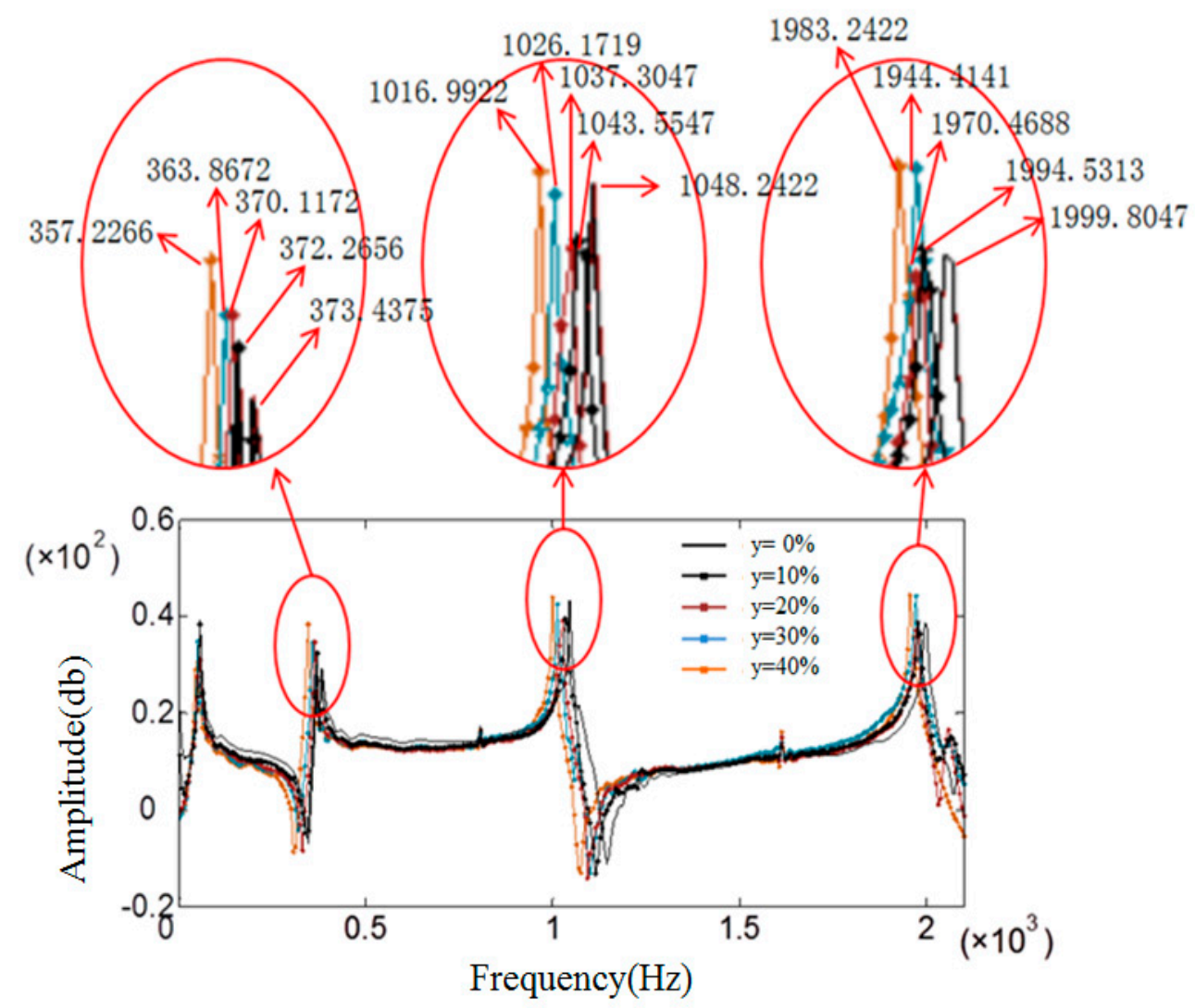

Figure 13. Frequency spectra of cantilever beam with a crack of the depth, $y$, increasing from $10 \%$ to $40 \%$ at the step of $10 \%$ at the location of $x=3.3 \%$.

Table 3. Three crack cases with identified crack location and depth.

\begin{tabular}{ccccccc}
\hline & \multicolumn{3}{c}{ Crack Location } & \multicolumn{3}{c}{ Crack Depth } \\
\cline { 2 - 6 } & Actual & Identified & Error & Actual & Identified & Error \\
\hline Case 1 & $3.30 \%$ & $2.95 \%$ & $3.39 \%$ & $10 \%$ & $9.75 \%$ & $2.5 \%$ \\
Case 2 & $46.70 \%$ & $46.00 \%$ & $1.50 \%$ & $20 \%$ & $20.30 \%$ & $1.5 \%$ \\
Case 3 & $86.70 \%$ & $83.85 \%$ & $3.10 \%$ & $40 \%$ & $39.00 \%$ & $2.5 \%$ \\
\hline
\end{tabular}

\subsubsection{Case 2 and Case 3}

The above procedure is implemented to identify cracks for Case 2 and Case 3 . The eventual intersection regions are presented in Figures 14 and 15, respectively. The identified locations and depths for Case 2 and Case 3 are shown in Table 3. As seen from Table 3, the error of identification for crack location is less than $3.4 \%$ and that for crack depth is less than $2.5 \%$. 


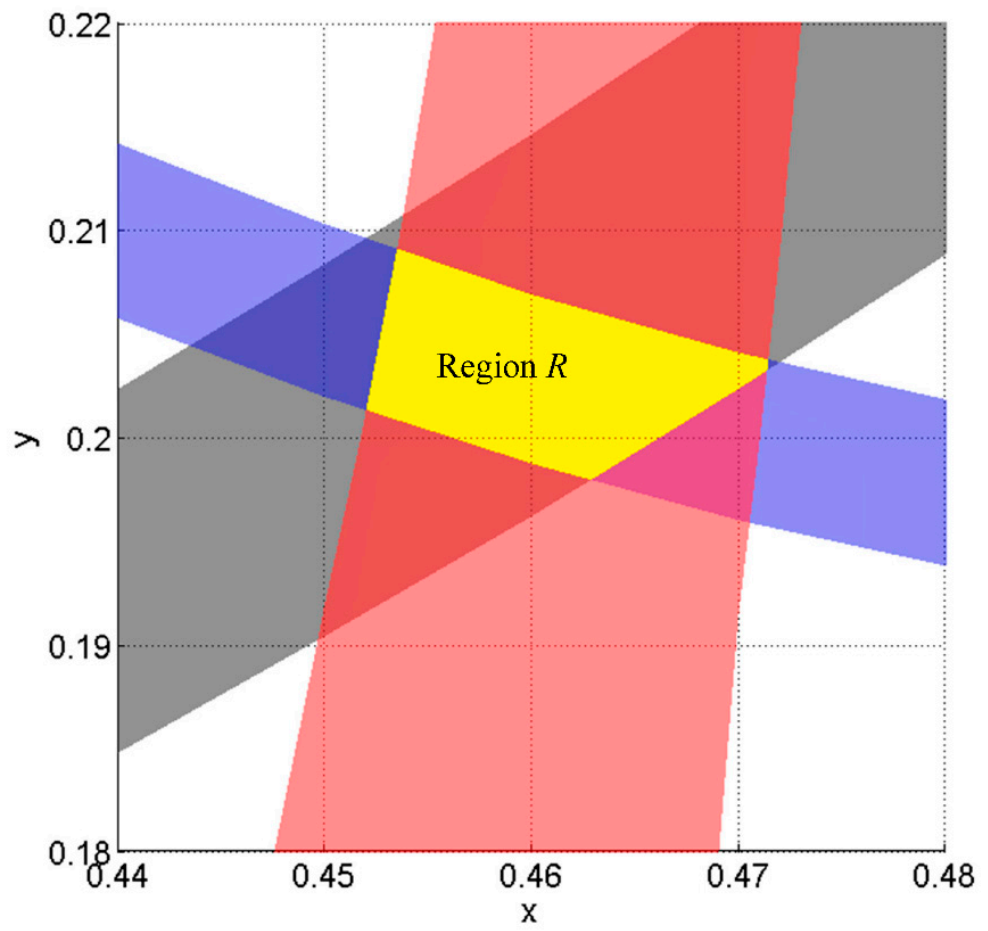

Figure 14. Intersection region R for Case 2.

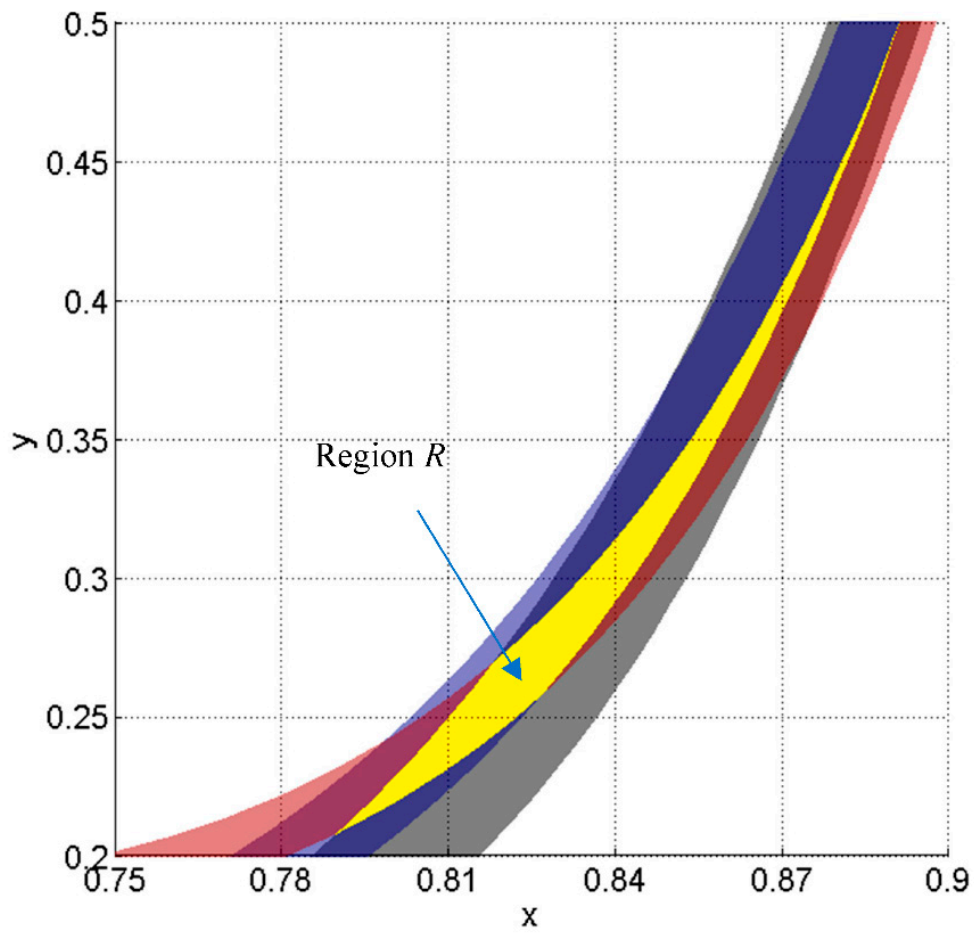

Figure 15. Intersection region $\mathrm{R}$ for Case 3.

These three cases verified the frequency contour-strip method to detect the cracks effectively, which were located near the fixed end, in the central part and near the free end of the beams, respectively. These cases illustrate the availability of the proposed method to detect the crack with about $3.7 \%$ errors of the testing frequencies. According to the research of Hiroshi Tada et al., the error of the damage correction factor in this research shown as Equation $(7 \mathrm{~b})$ is less than $0.5 \%$ for all crack depths. That is to say, the frequency contourstrip method is applicable, when the vibration equation can characterize the cracked beam. 
In fact, when the crack is very small, the frequency contour-strip will be wide, even though the error of the testing frequency is small. The main reason is that the frequency is not sensitive to the little small crack of the beam. Therefore, the relative error of the proposed method may become bigger when the crack is much smaller.

\section{Conclusions}

The developed frequency contour-strip method, from the theoretical perspective, is an extension of the frequency contour-line method. From the technological perspective, the frequency contour-strip method functions as a promotion of the frequency contour-line method. The developed method enjoys two predominant features in characterizing damage in beam-type structures: instantly establishing eigen-frequency panorama and accommodation of noise effect, substantially overcoming the limitations of existing frequency contourline method. The experimental results show that the frequency contour-strip method can characterize damage in beam-type structures with more efficiency, greater accuracy, and stronger robustness against noise. Noticeably, the proposed method is developed based on the eigen-frequency, a concept within the scope of linear dynamics. Therefore, the viability of the proposed method is confined to linear or approximately linear dynamic systems. Future following work will address the following issues: (i) validation of the proposed method in beam-type structures under other boundary conditions; (ii) extension of the proposed method for multiple damage detection in beam-type structures; (iii) extension of the proposed method to structures with more complex geometries; (iv) extension of the proposed method to real-time online damage identification in structures.

Author Contributions: Methodology, J.P., H.X., M.C. and H.J.; validation, J.P., H.J., T.D., D.S. and M.C.; data analysis, J.P., X.Z., D.S. and T.D.; resources, M.C. and D. S.; writing-original draft preparation, J.P. and X.Z.; Experiment: H.J., T.D. and J.P.; writing-review and editing, H.X., T.D. and D.S. All authors read, discussed, and commented on the manuscript. All authors have read and agreed to the published version of the manuscript.

Funding: This work is supported by the Key R\&D Project of Jiangxi Science and Technology Department (No. 20192BBH80021), National defense fundamental scientific research project (XXXX2018204B011), the Nanjing Science and Technology Project (No. 202002014), and the Fundamental Research Funds for the Central Universities of China (No. 2019B52914).

Institutional Review Board Statement: Not applicable.

Informed Consent Statement: Not applicable.

Conflicts of Interest: The authors declare no conflict of interest. The funders had no role in the design of the study; in the collection, analyses, or interpretation of data; in the writing of the manuscript, or in the decision to publish the results.

\section{References}

1. Dimarogonas, A.D. Vibration of cracked structures: A state of the art review. Eng. Fract. Mech. 1996, 55, 831-857. [CrossRef]

2. Cui, L.; Xu, H.; Ge, J.; Cao, M.; Xu, Y.; Xu, W.; Sumarac, D. Use of Bispectrum Analysis to Inspect the Non-Linear Dynamic Characteristics of Beam-Type Structures Containing a Breathing Crack. Sensors 2021, 21, 1177. [CrossRef]

3. Gounaris, G.; Dimarogonas, A. A finite element of a cracked prismatic beam for structural analysis. Comput. Struct. 1988, 28, 309-313. [CrossRef]

4. Huang, M.-S.; Gül, M.; Zhu, H.-P. Vibration-Based Structural Damage Identification under Varying Temperature Effects. J. Aerosp. Eng. 2018, 31, 04018014. [CrossRef]

5. Cawley, P.; Adams, R.D. Defect Location in Structures by a Vibration Technique; Ninth World Conference on Non-Destructive Testing: St. Louis, MO, USA, 1979.

6. Christides, S.; Barr, A. One-dimensional theory of cracked Bernoulli-Euler beams. Int. J. Mech. Sci. 1984, 26, 639-648. [CrossRef]

7. Kordestani, H.; Zhang, C.; Shadabfar, M. Beam Damage Detection under a Moving Load Using Random Decrement Technique and Savitzky-Golay Filter. Sensors 2019, 20, 243. [CrossRef] [PubMed]

8. Al-Hababi, T.; Cao, M.; Saleh, B.; Alkayem, N.F.; Xu, H. A Critical Review of Nonlinear Damping Identification in Structural Dynamics: Methods, Applications, and Challenges. Sensors 2020, 20, 7303. [CrossRef] [PubMed]

9. He, W.-Y.; He, J.; Ren, W.-X. Damage localization of beam structures using mode shape extracted from moving vehicle response. Measurement 2018, 121, 276-285. [CrossRef] 
10. Sha, G.; Radzienski, M.; Soman, R.; Cao, M.; Ostachowicz, W.; Xu, W. Multiple damage detection in laminated composite beams by data fusion of Teager energy operator-wavelet transform mode shapes. Compos. Struct. 2020, 235, 111798. [CrossRef]

11. Qiao, P.; Lu, K.; Lestari, W.; Wang, J. Curvature mode shape-based damage detection in composite laminated plates. Compos. Struct. 2007, 80, 409-428. [CrossRef]

12. Shahsavari, V.; Chouinard, L.; Bastien, J. Wavelet-based analysis of mode shapes for statistical detection and localization of damage in beams using likelihood ratio test. Eng. Struct. 2017, 132, 494-507. [CrossRef]

13. Roy, K.; Ray-Chaudhuri, S. Fundamental mode shape and its derivatives in structural damage localization. J. Sound Vib. 2013, 332, 5584-5593. [CrossRef]

14. Kawiecki, G. Modal damping measurement for damage detection. Smart Mater. Struct. 2001, 10, 466-471. [CrossRef]

15. Curadelli, R.O.; Riera, J.D.; Ambrosini, D.; Amani, M.G. Damage detection by means of structural damping identification. Eng. Struct. 2008, 30, 3497-3504. [CrossRef]

16. Frizzarin, M.; Feng, M.Q.; Franchetti, P.; Soyoz, S.; Modena, C. Damage detection based on damping analysis of ambient vibration data. Struct. Control. Health Monit. 2008, 17, 368-385. [CrossRef]

17. Cao, M.; Sha, G.; Gao, Y.; Ostachowicz, W. Structural damage identification using damping: A compendium of uses and features. Smart Mater. Struct. 2016, 26, 043001. [CrossRef]

18. Xiang, J.; Liang, M. Wavelet-Based Detection of Beam Cracks Using Modal Shape and Frequency Measurements. Comput. Civ. Infrastruct. Eng. 2012, 27, 439-454. [CrossRef]

19. Shokrani, Y.; Dertimanis, V.K.; Chatzi, E.N.; Savoia, M.N. On the use of mode shape curvatures for damage localization under varying environmental conditions. Struct. Control Health Monit. 2018, 25, e2132. [CrossRef]

20. Bai, R.; Cao, M.; Su, Z.Q.; Ostachowicz, W.; Xu, H. Aw Fractal Dimension Analysis of Higher-Order Mode Shapes for Damage Identification of Beam Structures. Math. Probl. Eng. 2012, 2012, 454568. [CrossRef]

21. Bai, R.B.; Ostachowicz, W.; Cao, M.S.; Su, Z. Crack detection in beams in noisy conditions using scale fractal dimension analysis of mode shapes. Smart Mater. Struct. 2014, 23, 065014. [CrossRef]

22. Salawu, O. Detection of structural damage through changes in frequency: A review. Eng. Struct. 1997, 19, 718-723. [CrossRef]

23. Owolabi, G.; Swamidas, A.; Seshadri, R. Crack detection in beams using changes in frequencies and amplitudes of frequency response functions. J. Sound Vib. 2003, 265, 1-22. [CrossRef]

24. Patil, D.; Maiti, S. Experimental verification of a method of detection of multiple cracks in beams based on frequency measurements. J. Sound Vib. 2005, 281, 439-451. [CrossRef]

25. Lin, R.-J.; Cheng, F.-P. Multiple crack identification of a free-free beam with uniform material property variation and varied noised frequency. Eng. Struct. 2008, 30, 909-929. [CrossRef]

26. Sha, G.; Radzieński, M.; Cao, M.; Ostachowicz, W. A novel method for single and multiple damage detection in beams using relative natural frequency changes. Mech. Syst. Signal Process. 2019, 132, 335-352. [CrossRef]

27. Sha, G.; Cao, M.; Radzieński, M.; Ostachowicz, W. Delamination-induced relative natural frequency change curve and its use for delamination localization in laminated composite beams. Compos. Struct. 2019, 230, 111501. [CrossRef]

28. Khatir, S.; Dekemele, K.; Loccufier, M.; Khatir, T.; Wahab, M.A. Crack identification method in beam-like structures using changes in experimentally measured frequencies and Particle Swarm Optimization. Comptes Rendus Mécanique 2018, 346, 110-120. [CrossRef]

29. Dahak, M.; Touat, N.; Kharoubi, M. Damage detection in beam through change in measured frequency and undamaged curvature mode shape. Inverse Probl. Sci. Eng. 2019, 27, 89-114. [CrossRef]

30. Banerjee, A.; Panigrahi, B.; Pohit, G. Crack modelling and detection in Timoshenko FGM beam under transverse vibration using frequency contour and response surface model with GA. Nondestruct. Test. Eval. 2015, 31, 142-164. [CrossRef]

31. Yang, C.; Zhang, Z.; Nong, S.; Zhu, C. Analytical and experimental investigation on eigenfrequency-based damage diagnosis of cantilever beam. J. Vibroeng. 2016, 18, 5114-5126. [CrossRef]

32. Li, B.; Chen, X.; He, Z. Three-steps-meshing based multiple crack identification for structures and its experimental studies. Chin. J. Mech. Eng. 2013, 26, 400-405. [CrossRef]

33. Mazanoglu, K.; Sabuncu, M. A frequency based algorithm for identification of single and double cracked beams via a statistical approach used in experiment. Mech. Syst. Signal Process. 2012, 30, 168-185. [CrossRef]

34. Chen, X.F.; Li, B.; He, Y.M.; He, Z.J. Second Generation Wavelet Finite Element and Rotor Cracks Quantitative Identification Method. Chin. J. Mech. Eng. 2010, 23, 195-199. [CrossRef]

35. Cao, M.S.; Song, X.C.; Xu, W.; Su, Z.Q. Performance assessment of natural frequencies in characterizing cracks in beams in noisy conditions. J. Vibroeng. 2014, 16, 1010-1021.

36. Kalanad, A.; Rao, B.N. Detection of crack location and size in structures using improved damaged finite element. IOP Conf. Series: Mater. Sci. Eng. 2010, 10, 012054. [CrossRef]

37. Swamidas, A.S.J.; Yang, X.; Seshadri, R. Identification of Cracking in Beam Structures Using Timoshenko and Euler Formulations. J. Eng. Mech. 2004, 130, 1297-1308. [CrossRef]

38. Yu, Z.; Chu, F. Identification of crack in functionally graded material beams using the p-version of finite element method. J. Sound Vib. 2009, 325, 69-84. [CrossRef]

39. Lakshmanan, N.; Raghuprasad, B.; Gopalakrishnan, N.; Sathishkumar, K.; Murthy, S. Detection of contiguous and distributed damage through contours of equal frequency change. J. Sound Vib. 2010, 329, 1310-1331. [CrossRef] 
40. Dong, G.; Chen, J.; Zou, J. Parameter identification of a rotor with an open crack. Eur. J. Mech. A/Solids 2004, 23, 325-333. [CrossRef]

41. Liu, W.G.; Guo, L.Q.; He, H.L.; Yan, L. A damage identification method of a breathing cracked beam by natural frequency. China Mech. Eng. 2017, 28, 702-707, 713.

42. Tada, H.; Paris, P.C.; Irwin, G.R. Stress Analysis of Cracks Handbook, 3rd ed.; Tada, H., Ed.; ASME Press: New York, NY, USA, 1973.

43. Tada, H.; Paris, P.C.; Irwin, G.R. The Stress Analysis of Cracks Handbook, 2nd ed.; Paris Productions Incorporated: Paris, France, 1985.

44. Peng, J.Y.; Cao, M.S.; Xia, N.; Chen, J.G. Natural Frequency Spectra of Cracked Beams for Dynamic Property Characterization. Appl. Mech. Mater. 2013, 275-277, 247-251. [CrossRef]

45. Nandwana, B.; Maiti, S. Detection of the location and size of a crack in stepped cantilever beams based on measurements of natural frequencies. J. Sound Vib. 1997, 203, 435-446. [CrossRef] 